\title{
SUBOPTIMUM MAXIMUM LIKELIHOOD ESTIMATION OF STRUCTURAL PARAMETERS FROM MULTIPLE-EXCITATION VIBRATION DATA
}

\author{
J.E. Lee ${ }^{1}$, and, S.D. Fassois ${ }^{2}$
}

Report UM-MEAM-90-03

(C)1990 by J.E. Lee and S.D. Fassois All rights reserved.

\footnotetext{
1 Graduate Research Assistant, Mechanical Engineering and Applied Mechanics

2 Assistant Professor, Mechanical Engineering and Applied Mechanics
} 



\title{
SUBOPTIMUM MAXIMUM LIKELIHOOD ESTIMATION OF STRUCTURAL PARAMETERS \\ FROM MULTIPLE-EXCITATION VIBRATION DATA
}

\author{
J.E. Lee* and S.D. Fassois** \\ Department of Mechanical Engineering \\ and Applied Mechanics \\ The University of Michigan \\ Ann Arbor, MI 48109-2125
}

- Research Assistant, Mechanical Engineering and Applied Mechanics

** Asciatant Profeseor, Mechanical Engineering and Applied Mechanica 


\section{ABSTRACT}

In this paper a highly-effective, multiple-excitation, and stochastic, structural dynamics identification approach is introduced. The proposed approach is based on the newly developed (Fassois and Lee, 1988) Suboptimum Maximum Likelihood (SML) discrete estimation algorithm, systematic and efficient modeling strategy and model validation procedures, as well as accurate modal parameter extraction that is consistent with the employed model structure and excitation signal forms. This approach overcomes many major drawbacks of the currently available techniques, as it is shown to, unlike deterministic methods, offer consistent modal parameter estimates and high accuracy and resolution from noise-corrupted data records, while, unlike alternative stochastic methods, also featuring guaranteed algorithmic stability, complete elimination of the wrong convergence/erroneous estimated model problem, and very modest computational complexity.

The effectiveness of the proposed approach is verified through numerical simulations with structural models characterized by well-separated and closely-spaced vibrational modes, with particular emphasis placed on the achievable accuracy and resolution at different noise levels, effectiveness with short data records, and the efficiency of the modeling strategy and model validation procedures. Comparisons with the classical frequency domain method are also made, whereas the drawbacks of deterministic approaches are illustrated through comparisons with the Eigensystem Realization Algorithm (ERA). Experimental results, where the proposed approach is used for the modal analysis of a flexible beam from laboratory data, are also presented. 


\section{INTRODUCTION}

Time-domain structural parameter identification methods have received significant attention in recent years as they have been shown to overcome many of the drawbacks of classical frequency-domain approaches and offer improved accuracy and resolution even with short data records and at significantly reduced computational and memory storage requirements (Ibrahim and Mikulcik, 1973; Vold and Rocklin, 1982; Juang and Pappa, 1985; Leuridan et al., 1986; Snoeys et al., 1987; Fassois et al., 1989a).

Most of the available time domain methods are, however, inherently deterministic, and therefore incapable of effectively dealing with noise-corrupted experimental data. Indeed, it is a well-known fact that the quality of structural parameter estimates obtained through such methods decreases drastically as the noise-to-signal (N/S) ratio ceases to be negligible, and a number of modes may in fact be completely impossible to estimate (Davies and Hammond, 1984; Fritzen, 1986; Ben Mrad and Fassois, 1989). In a commonly attempted remedy for this problem, significant model order overdetermination is used (Braun and Ram, 1987; Lee and Tretheway, 1988). Model overdetermination may, indeed, allow for the estimation of more vibrational modes than otherwise possible, but it is also characterized by its own limitations, namely the significantly increased computational and memory storage requirements (due to the excessively oversized models typically used), and the very severe difficulties in the assessment of the required model order and the final distinction between the estimated false ("computational") and actual ("structural") modes. In addition, the achievable resolution may remain poor, and, as it has been observed, the "identification scatter" increases (and hence accuracy decreases) for all modes as the order of the model increases beyond its true value (Juang and Pappa, 1986). 
As a consequence, stochastic methods, capable of effectively accounting for the effects of noise and yielding accurate modal parameter estimates at realistic N/S ratios, are required. One such method, refered to as Dynamic Data System (DDS), was proposed by Kim et al. (1984) based on nonlinear Maximum Likelihood (ML) estimation; an approach known to yield statistically optimal (consistent, efficient, and asymptotically Gaussian) estimates under mild assumptions ( $\AA$ ström, 1980). The highly nonlinear nature of the estimation algorithm, however, makes the DDS method computationally expensive, and also vulnerable to a number of problems, such as the need for initial guess parameter values, the occurrence of algorithmic instabilities (and as a consequence failure of the method to provide a model), and the possibility of convergence to a completely erroneous model; a phenomenon due to the possible existence of a number of local minima in the negative log-likelihood function (Söderström, 1975). A stochastic linear method, that offers consistent estimates and improved modal parameter extraction at a reduced computational complexity, was recently proposed by Fassois et al. (1989a). Although reduced, the number of computations may be still significant, as several iterations in two estimation modes are required. In addition, this method has not resolved the algorithmic instability and wrong convergence problems from which it also suffers.

It becomes thus apparent that for the development of a reliable and effective stochastic approach, the following limitations and drawbacks of the currently available stochastic structural dynamics identification methods need to be overcome: (a) The occurrence of algorithmic instabilities, (b) possible convergence to a completely erroneous model; a phenomenon associated with the existence of local minima in the identification criterion, (c) high computational complexity; a consequence of using nonlinear estimation schemes and/or highly biased initial 
parameter estimates, (d) the need for initial guess parameter values. In addition, issues related to efficient model order determination, model validation, and accurate modal parameter extraction, need to be further addressed and effective procedures developed.

In the present paper these problems are addressed, and a stochastic, time-domain, multipleexcitation, structural dynamics identification approach that effectively overcomes the drawbacks of deterministic techniques as well as the aforementioned difficulties of existing stochastic methods, is introduced. The proposed approach is based on a novel, Suboptimum Maximum Likelihood (SML) discrete estimation algorithm recently introduced by Fassois and Lee (1988), a systematic modeling strategy that incorporates statistical decision theory criteria and is very effective in selecting the appropriate model orders without any need for subjective judgement, a model validation procedure that is based on the predictive ability of the estimated model and effectively controls model acceptance, and a modal parameter extraction scheme that unlike those of alternative methods is consistent with the model structure and excitation signal form employed. The main features and advantages of the proposed approach may be specifically summarized as follows:

- Statistically consistent modal parameter estimation in the presence of correlated or uncorrelated noise, and very modest (significantly smaller than that of alternative stochastic methods) computational complexity.

- Mathematically guaranteed algorithmic stability. In contrast to alternative stochastic methods, the proposed approach is characterized by mathematically guaranteed stability.

- Complete elimination of the wrong convergence/erroneous estimated model problem. 
Unlike alternative methods, the proposed approach yields a unique model that is shown to asymptotically converge to the true structural model.

- No need for initial guess parameter values and minimal operator intervention.

- Effective modeling strategy and model validation procedures.

- Accurate modal parameter extraction that is consistent with the employed model structure and excitation signal form.

- Estimation based on multiple-excitation data; a feature that significantly contributes in achieving higher quality estimates and drastically reducing the data acquisition and processing time requirements.

The performance of the proposed approach is carefully evaluated with simulated and experimental vibration data. For this purpose structural models characterized by well-separated and closely-spaced modes are employed, and particular emphasis is placed on the achievable estimation accuracy and resolution at different $\mathrm{N} / \mathrm{S}$ ratios, the effectiveness of the approach with "short" data records, its rate of convergence, and the efficiency of the modeling strategy and model validation procedures; the last one being an issue that despite its significance is often neglected in the literature. In addition, comparisons with the well-known classical Frequency Domain Method (FDM) are made, and the inadequacy of deterministic approaches in dealing with noise-corrupted data illustrated through comparisons with the Eigensystem Realization Algorithm (ERA) (Juang and Pappa, 1985).

The paper is organized as follows: The proposed structural dynamics identification approach, along with its main properties, the modeling strategy, the model validation, and the modal parameter extraction procedures, is presented in Section 2. Its effectiveness and 
specific performance characteristics are examined in Section 3 by using simulated vibration data and comparisons with the classical frequency domain and ERA methods. In Section 4 the proposed approach is used for the modal analysis of a flexible beam from experimental data, and the conclusions are finally summarized in Section 5.

\section{THE IDENTIFICATION APPROACH}

In this section the stochastic structural dynamics identification problem is formulated, and the proposed multiple-excitation approach presented. The proposed approach utilizes special forms of Autoregressive Moving-Average with eXogeneous inputs (ARMAX) models that fully and distinctly represent both the structural and noise dynamics, and is based on a novel Suboptimum Maximum Likelihood (SML) estimation algorithm, efficient modeling strategy and model validation procedures, and a modal parameter extraction scheme that is consistent with the particular model structure and excitation signal form employed.

\subsection{ARMAX Representation of Stochastic Structural Systems}

The dynamics of a linear, viscously-damped, $n$ degree-of-freedom structural system may be represented by a vector differential equation of the form :

$$
\mathbf{M} \cdot \ddot{\mathbf{v}}(t)+\mathbf{C} \cdot \dot{\mathbf{v}}(t)+\mathbf{K} \cdot \mathbf{v}(t)=\mathbf{f}(t)
$$

or equivalently :

$$
\mathrm{V}(s)=\left[\mathbf{M} \cdot s^{2}+\mathbf{C} \cdot s+\mathbf{K}\right]^{-1} \cdot \mathbf{F}(s)=\mathbf{G}(s) \cdot \mathbf{F}(s)
$$

where $\mathbf{M}, \mathbf{C}, \mathbf{K}$ represent the mass, viscous damping, and stiffness matrices, respectively, $\{\mathbf{f}(t)\}$ the force excitation (vector) signal, $\{\mathbf{v}(t)\}$ the resulting vibration displacement (vector) signal, $s$ the Laplace Transform variable, and $\mathbf{G}(s)$ the corresponding transfer matrix. The 
stochastic structural dynamics identification problem may be then posed as follows : "Given uniformly-sampled excitation $\{f[t]\}_{t=1}^{N}$ and noise-corrupted response $\{y[t]\}_{t=1}^{N}$ data, where :

$$
\mathbf{y}[t]=\mathbf{v}[t]+\mathbf{n}[t]
$$

with $\{\mathbf{n}[t]\}$ representing a zero-mean stationary stochastic noise (vector) process which is uncross-correlated with $\{\mathbf{f}[t]\}$, estimate a model of the form (1), or equivalently its set of natural frequencies, damping factors, and mode shapes, that characterizes the structure under study".

The approach taken in this paper for the solution of this problem is based on discrete-time identification combined with an appropriate discrete-to-continuous dynamic system transformation for modal parameter extraction. By using the reciprocity assumption, estimation of the discrete-time "equivalent" of just one row of (2) is sufficient for modal parameter estimation. That, combined with (3) gives the following system equation :

$$
y[t]=\sum_{i=1}^{n} \frac{B_{i}^{\circ}(B)}{A^{\circ}(B)} \cdot f_{i}[t]+n[t]
$$

where $\{y[t]\},\{n[t]\}$ represent the $\mathbf{j}$-th scalar component of $\{\mathbf{y}[t]\},\{\mathbf{n}[t]\}$, respectively, $\left\{f_{i}[t]\right\}$ the sampled version of the $\mathrm{i}$-th component of $\{\mathbf{f}(t)\}$, and $A^{\circ}(B), B_{i}^{\circ}(B)(1 \leq i \leq n)$ polynomials in the backshift operator $B(B \cdot y[t] \triangleq y[t-1])$, of the form:

$$
\begin{aligned}
& A^{\circ}(B) \triangleq 1+a_{1}^{\circ} \cdot B+\cdots+a_{2 n}^{\circ} \cdot B^{2 n} \\
& B_{i}^{\circ}(B) \triangleq b_{0, i}^{\circ}+b_{1, i}^{\circ} \cdot B+\cdots+b_{m_{i}, i}^{\circ} \cdot b^{m_{i}} \quad(1 \leq i \leq n)
\end{aligned}
$$

with $A^{\circ}(B)$ being strictly minimum phase (for stability) and relatively prime to $B_{i}^{\circ}(B) \quad(1 \leq$ $i \leq n)$

By further assuming that the noise $\{n[t]\}$ is characterized by a rational spectral density, the spectral factorization theorem ( $₫$ ström, 1970) implies that the structural system equation 
(4) may be put into the standard multiple-input $\operatorname{ARMAX}\left(2 n, m_{1}, m_{2}, \ldots, m_{n}, l\right)$ form:

$$
A^{\circ}(B) \cdot y[t]=\sum_{i=1}^{n} B_{i}^{\circ}(B) \cdot f_{i}[t]+C^{\circ}(B) \cdot w[t]
$$

with $C^{\circ}(B)$ representing a strictly minimum phase and monic polynomial of the form:

$$
C^{\circ}(B) \triangleq 1+c_{1}^{\circ} \cdot B+\cdots+c_{i}^{\circ} \cdot B^{l}
$$

which is assumed to be relatively prime to $A^{\circ}(B)$ and of (possibly infinite) order $l$, whereas $\{w[t]\}$ represents an innovations (zero mean and uncorrelated) sequence which is uncrosscorrelated with $\left\{f_{i}[t]\right\}(1 \leq i \leq n)$ and has variance $\left(\sigma_{w}^{\circ}\right)^{2}$. Within the context of the proposed approach a special form of the multiple-input ARMAX model of Eq. (6), in which $m_{1}=$ $m_{2}=\ldots=m_{n}=2 n-1$ and no time-delay exists $\left(b_{0, i} \neq 0\right.$ for $\left.1 \leq i \leq n\right)$, will be in fact used (see subsections 2.3.1, 2.3.3 and the justifications therein). This ARMAX representation fully and distinctly represents both the structural $\left[B_{i}^{o}(B) / A^{\circ}(B)\right.$ for $\left.i=1,2, \ldots, n\right]$ and noise $\left[C^{\circ}(B) / A^{\circ}(B)\right]$ dynamics, and can be effectively used for modal parameter estimation from noise-corrupted data.

\subsection{The Discrete Parameter Estimation Algorithm}

Parameter estimation of stochastic multiple-input ARMAX models of the form [compare with (6)]:

$$
A(B, \theta) \cdot y[t]=\sum_{i=1}^{n} B_{i}(B, \theta) \cdot f_{i}[t]+C(B, \theta) \cdot e[t / \theta]
$$

where $\theta$ represents the unknown parameter vector :

$$
\theta \triangleq\left[\operatorname{coef} A, \operatorname{coef} B_{1}, \cdots, \operatorname{coef} B_{n}, \operatorname{coef} C\right]^{T}
$$

$e[t / \theta]$ the one-step ahead prediction error associated with the model, and the autoregressive (AR) $A(B, \theta)$, moving-average (MA) $B_{i}(B, \theta)$, and exogenous (X) $C(B, \theta)$ polynomials being 
of the form (5a), (5b), and (7), respectively, is the first step required for structural dynamics identification.

Within the context of the proposed approach this is achieved by using the Suboptimum Maximum Likelihood (SML) estimation algorithm recently introduced in a general context by Fassois and Lee (1988). The SML is a novel, multi-stage, highly reliable and computationally efficient estimation algorithm that attempts to approximate the ML solution while overcoming its well-known drawbacks. This is accomplished by using a quadratic approximation of the negative log-likelihood function about an initial estimate in the MA parameter subspace, which is then minimized to yield the AR and exogenous parameter estimates. The final MA parameters are then obtained by using parameter estimates of a related ARX process and fundamental ARMAX process properties. A brief account of the stages of the SML algorithm for ARMAX process parameter estimation is given in the sequel.

Stage 1: ARX estimation.

A multiple-input ARX model of the form:

$$
H_{y}(B, \mathbf{h}) \cdot y[t]=\sum_{i=1}^{n} H_{x i}(B, \mathbf{h}) \cdot f_{i}[t]+e_{p}[t / \mathbf{h}]
$$

where $H_{y}(B, \mathrm{~h})$ and $H_{x i}(B, \mathrm{~h})$ represent p-th order polynomials of the form:

$$
\begin{aligned}
H_{y}(B, \mathbf{h}) & \triangleq 1+h_{y 1} \cdot B+\cdots+h_{y p} \cdot B^{p} \\
H_{x i}(B, \mathbf{h}) & \triangleq h_{x i 0}+h_{x i 1} \cdot B+\cdots+h_{x i p} \cdot B^{p} \quad(1 \leq i \leq n)
\end{aligned}
$$

$\left\{e_{p}[t / h]\right\}$ the one-step ahead prediction error sequence associated with this model, and $\mathbf{h}$ the parameter vector :

$$
\mathrm{h} \triangleq\left[h_{x 10} \cdots h_{x n 0} \vdots h_{y 1} h_{x 11} \cdots h_{x n 1} \vdots \cdots \vdots h_{y p} h_{x i p} \cdots h_{x n p}\right]^{T}
$$


is estimated. This is achieved through the "covariance-type" least-squares (LS) estimator:

$$
\hat{\mathbf{h}}=\left(\sum_{t=p+1}^{N} \psi_{h}[t] \cdot \psi_{h}^{T}[t]\right)^{-1} \cdot\left(\sum_{t=p+1}^{N} \psi_{h}[t] \cdot y[t]\right)
$$

where :

$$
\begin{array}{r}
\boldsymbol{\psi}_{h}[t] \triangleq\left[f_{1}[t] \cdots f_{n}[t] \vdots-y[t-1] f_{1}[t-1] \cdots f_{n}[t-1] \vdots \cdots \vdots\right. \\
\left.-y[t-p] f_{1}[t-p] \cdots f_{n}[t-p]\right]^{T}
\end{array}
$$

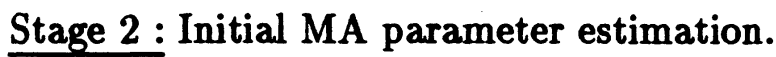

An initial MA parameter vector estimate is obtained as:

$$
\hat{\mathbf{c}}_{0}=\hat{\hat{c}}_{00}^{-1} \cdot\left[\begin{array}{llll}
\hat{c}_{01} & \hat{\boldsymbol{c}}_{02} & \cdots & \hat{\underline{c}}_{01}
\end{array}\right]^{T}
$$

where:

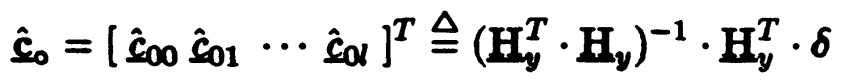

$$
\begin{aligned}
& \delta \triangleq\left[\begin{array}{lllll}
1 & 0 & 0 & \cdots & 0
\end{array}\right] \in \Re^{p+l-2 n+1}
\end{aligned}
$$

and $\mathbf{H}_{y}$ being a $(p+l-2 n+1) \times(l+1)$ matrix of the ARX parameter estimates of the form:

$$
\mathbf{H}_{y} \triangleq\left[\begin{array}{cccc}
\hat{h}_{y(2 n)} & & & \\
\hat{h}_{y(2 n+1)} & \hat{h}_{y(2 n)} & 0 & \\
\cdot & \hat{h}_{y(2 n+1)} & \ddots & \\
\vdots & & \ddots & \hat{h}_{y(2 n)} \\
\vdots & & & \hat{h}_{y(2 n+1)} \\
\hat{h}_{y p} & & & \\
& \hat{h}_{y p} & & \\
& 0 & \ddots & \hat{h}_{y p}
\end{array}\right]
$$


Stage 3: AR and exogenous parameter estimation.

The AR and exogenous parameters are obtained by estimating an ARX model of the form:

$$
A(B, \phi) \cdot y^{F}\left[t / \hat{\mathbf{c}}_{\mathrm{o}}\right]=\sum_{i=1}^{n} B_{i}(B, \phi) \cdot f_{i}^{F}\left[t / \hat{\mathbf{c}}_{\mathrm{o}}\right]+e^{\prime}\left[t / \phi, \hat{\mathbf{c}}_{\mathrm{o}}\right]
$$

where $\phi$ represents the combined AR/exogenous parameter vector:

$$
\phi \triangleq\left[\operatorname{coef} A, \operatorname{coef} B_{1}, \cdots, \operatorname{coef} B_{n}\right]^{T}
$$

$\hat{\mathbf{c}}_{\mathrm{o}}$ the previously obtained initial MA parameter estimate, $\left\{e^{\prime}\left[t / \phi, \hat{\mathrm{c}}_{\mathrm{o}}\right]\right\}$ the one-step ahead prediction error sequence associated with the model structure of Eq. (19), and :

$$
\begin{aligned}
y^{F}\left[t / \hat{\mathbf{c}}_{o}\right] & \triangleq \hat{C}_{o}^{-1}(B) \cdot y[t] \\
f_{i}^{F}\left[t / \hat{\mathbf{c}}_{\mathrm{o}}\right] & \triangleq \hat{C}_{o}^{-1}(B) \cdot f_{i}[t] \quad(1 \leq i \leq n)
\end{aligned}
$$

where $\hat{C}_{0}^{-1}(B)$ represents the estimate of the MA polynomial obtained in Stage 2. This is accomplished through the "covariance-type" LS estimator:

$$
\hat{\boldsymbol{\phi}}=\left(\sum_{t=q+1}^{N} \psi[t] \cdot \psi^{T}[t]\right)^{-1} \cdot\left(\sum_{t=q+1}^{N} \psi[t] \cdot y^{F}\left[t / \hat{\mathbf{c}}_{\mathrm{o}}\right]\right)
$$

with $q \triangleq \max \left(2 n, m_{1}, m_{2}, \cdots, m_{n}\right)$ and:

$$
\begin{array}{r}
\psi[t] \triangleq\left[-y^{F}\left[t-1 / \hat{\mathbf{c}}_{\mathrm{o}}\right] \cdots-y^{F}\left[t-2 n / \hat{\mathbf{c}}_{\mathrm{o}}\right]: f_{1}^{F}\left[t / \hat{\mathbf{c}}_{\mathrm{o}}\right] \cdots f_{1}^{F}\left[t-m_{1} / \hat{\mathbf{c}}_{\mathrm{o}}\right] \vdots \cdots \vdots\right. \\
\left.f_{n}^{F}\left[t / \hat{\mathbf{c}}_{\mathrm{o}}\right] \cdots f_{n}^{F}\left[t-m_{n} / \hat{\mathbf{c}}_{\mathrm{o}}\right]\right]^{T}
\end{array}
$$

Stage 4 : Final MA parameter and innovations sequence estimation.

The final MA parameter estimates are obtained from the following set of linear equations:

$$
\sum_{k=0}^{j} \hat{c}_{k} \cdot \hat{h}_{y(j-k)}=\hat{a}_{j} \quad(j=1,2, \cdots, l)
$$


where $\hat{a}_{j} \equiv 0$ for $j>2 n$, and the innovations sequence is then estimated as:

$$
e[t / \hat{\boldsymbol{\theta}}]=C^{-1}(B, \hat{\boldsymbol{\theta}}) \cdot\left[A(B, \hat{\boldsymbol{\theta}}) \cdot y[t]-\sum_{i=1}^{n} B_{i}(B, \hat{\boldsymbol{\theta}}) \cdot f_{i}[t]\right]
$$

\section{Remarks:}

A few brief, but important, remarks regarding the Suboptimum Maximum Likelihood estimation algorithm are now in order:

(a) Under mild assumptions, the SML parameter estimates are strongly consistent, that is they converge to the true parameter vector as the number of data $N$ and the ARX order $p$ tend to infinity. This result may be precisely stated as follows (Fassois and Lee, 1988):

Proposition: Under the standard system assumptions of subsection 2.1, appropriate ergodicity and persistent excitation conditions for the excitation signals, and model orders that coincide with the actual system orders, the SML estimator is strongly consistent, that is:

$$
\begin{array}{r}
\hat{\theta}(N, p) \rightarrow \underline{\theta}(p) \quad \text { w.p. } 1 \text { as } N \rightarrow \infty \\
\underline{\theta}(p) \rightarrow \theta^{\circ} \text { as } p \rightarrow \infty \quad(p<<N)
\end{array}
$$

where $\theta(p)$ represents a deterministic vector that depends on $p, \theta^{\circ}$ the true parameter vector, and the symbol w.p.1 indicates convergence with probability 1 .

Furthermore, the algorithm is exclusively based on linear operations and the minimization of quadratic criteria, and hence the estimate $\hat{\theta}(N, p)$ is unique. Since $\hat{\theta}(N, p)$ was additionally shown to converge to the true parameter vector, it is evident that wrong convergence or convergence to a local minimum problems that are associated with alternative techniques, are eliminated eliminated.

(b) Unlike alternative approaches (such as the Maximum Likelihood or some Instrumental Variable variants), the SML algorithm offers mathematically guaranteed algorithmic stability! 
This is due to the fact that the initial MA parameter estimator (15) is, by construction, guaranteed to yield a strictly minimum phase $\hat{C}_{\circ}(B)$ polynomial (Fassois and Lee, 1988).

(c) The order $p$ of the ARX model (10) needs to be selected such that $2 n+l<p<<N$. Although the accurate determination of an adequate $p$ may be formally accomplished through statistical decision theory criteria such as the AIC (see subsection 2.3.1), it has been found that for structural dynamics problems a simple rule of thumb is to select $p$ in the range:

$$
p \cong(2.5 \sim 4) \times \max \left(2 n, m_{1}, \cdots, m_{n}, l\right)
$$

The specific value of $p$ does not seem to significantly affect estimation accuracy, provided of course that a sufficiently large value has been selected.

(d) Unlike some alternative approaches, the SML algorithm requires no initial guess parameter values.

(e) Finally, Stages 3 and 4 of the algorithm may be iterated until the resulting sequence of estimates of $\theta$ converges. This issue will be further examined and discussed in Sections 3 and 4.

\subsection{Modeling Strategy, Model Validation, and Modal Parameter Extraction}

\subsubsection{Modeling Strategy}

An efficient modeling strategy is a basic element of any effective structural dynamics identification method. The reason for this is that in practice neither the necessary number of degrees of freedom for an "adequate" structural representation, nor the required order of the MA (noise) polynomial, are a priori known.

In this work a well-defined, systematic and efficient strategy, that is based on fundamental properties of structural systems and utilizes a special form of multiple-input AR- 
MAX models, has been formulated. Its main steps may be outlined as follows: A sequence of ARMAX $(2 k, \underbrace{2 k-1, \cdots, 2 k-1}_{n \text { times }}, 2 k)$ models with no time-delay are successively fitted for $k=1,2,3, \cdots$, until a statistically adequate representation, say $\operatorname{ARMAX}\left(2 k^{*}, 2 k^{*}-\right.$ $\left.1, \cdots, 2 k^{*}-1,2 k^{*}\right)$ is achieved. A sequence of $\operatorname{ARMAX}\left(2 k^{*}, 2 k^{*}-1, \cdots, 2 k^{*}-1, l\right)$ models is subsequently fitted for $l=2 k^{*}-1,2 k^{*}-2, \cdots$, and the "best" representation, say $\operatorname{ARMAX}\left(2 k^{*}, 2 k^{*}-1, \cdots, 2 k^{*}-1, l^{*}\right)$, selected for $l \leq 2 k^{*}$. If $l^{*}<2 k^{*}$ this is adopted as the final adequate model and the procedure ends. Otherwise $\left(l^{*}=2 k^{*}\right)$ a new sequence of $\operatorname{ARMAX}\left(2 k^{*}, 2 k^{*}-1, \cdots, 2 k^{*}-1, l\right)$ models is successively fitted for $l=2 k^{*}+1,2 k^{*}+2, \cdots$, and the "best" representation, say ARMAX $\left(2 k^{*}, 2 k^{*}-1, \cdots, 2 k^{*}-1, l^{*}\right)$, selected for $l \geq 2 k^{*}$ as the final adequate model.

The reason for the selection and testing of even AR orders is simply due to the fact that structural systems are characterized by pairs of complex conjugate eigenvalues, each one corresponding to a single degree of freedom. On the other hand, the time-delay is set equal to zero and the exogenous orders are invariably selected to be equal to the AR order minus one, because this is the structure of the discrete transfer function obtained from a continuous-time system with arbitrary numerator dynamics when the impulse-invariance principle is used for modal parameter extraction (as is the case with the present approach, see subsection 2.3.3). Finally, the MA order is initially set equal to the AR order since the resulting noise model $\left[A^{-1}(B) \cdot C(B)\right]$ can adequately represent an uncorrelated sequence as well as a class of other processes. The exact MA order required, however depends upon the specific noise statistics, so that "fine-tuning" of this parameter is practically necessary. Values smaller than the AR order are then examined first, since experience with structural systems indicates that for relatively low noise levels the final adequate model often has an MA order that is equal or 
slightly smaller than the AR order. Nevertheless, higher orders may be also needed, and are therefore subsequently examined.

Finally, statistical adequacy is judged by means of Akaike's Information Criterion [AIC] (Akaike, 1974), according to which an $A R M A X(n a, n b, \ldots ., n b, n c)$ model is selected as statistically adequate if it minimizes the index:

$$
\operatorname{AIC}(n a, n b, n c)=N \cdot \ln \hat{\sigma}_{e}^{2}+2 \cdot(n a+n \cdot n b+n c)
$$

where $n a, n b, n c$, represent the AR, exogenous, and MA orders, $n$ the number of excitations (equal to that of degrees of freedom), and $\hat{\sigma}_{e}^{2}$ the estimated innovations variance.

\subsubsection{Model Validation}

Before the finally selected ARMAX model can be accepted as an accurate discrete-time representation of the actual structural and noise dynamics, it should successfully pass an appropriate model validation procedure. Within the context of the proposed approach, a two-phase procedure that concentrates on the predictive ability of the estimated model has been formulated and is implemented as follows:

Phase 1: The normalized autocorrelation function of the one-step ahead prediction error sequence associated with the estimated model (estimated innovations sequence) is evaluated as:

$$
\hat{\rho}[\tau]=\frac{\sum_{t}(e[t]-\bar{e})(e[t-\tau]-\bar{e})}{\sum_{t}(e[t]-\bar{e})^{2}}
$$

where $e[t]$ represents the one-step ahead prediction error at time $t$ estimated from Eq. (25) and $\bar{e}$ its sample mean value. For a model that accurately represents the true structural system and the noise affecting it, $\{e[t]\}$ must be uncorrelated. The uncorrelatedness of $\{e[t]\}$ 
is thus examined through the standard statistical check (Pandit and Wu, 1983) :

$$
|\hat{\rho}[\tau]|<\frac{2}{\sqrt{N}} \quad \forall \tau \neq 0
$$

and an estimated model passes Phase 1 if expression (30) is satisfied.

Phase 2: The predictive ability of the model is further evaluated by using a data set, refered to as the validation set, that is different from the estimation set used for model estimation. For this purpose a standard predictor corresponding to the estimated ARMAX model is built, and the measured vibration signal $\{y[t]\}$ in the validation set is compared to the model-predicted signal $\{\hat{y}[t / \hat{\theta}]\}$. A measure of discrepancy is then constructed as:

$$
Q=\frac{\sum_{t=N+1}^{N+N_{v}}(\hat{y}[t / \hat{\theta}])^{2}}{\sum_{t=N+1}^{N+N}(y[t])^{2}}
$$

where $N_{v}$ represents the total number of data in the validation set, and used in judging model performance. In this case a model is accepted as long as $Q$ is greater than a prespecified threshold value.

\subsubsection{Modal Parameter Extraction}

After the estimated ARMAX model has been validated, it is rewritten as:

$$
y[t]=\sum_{i=1}^{n} \frac{B_{i}(B, \hat{\theta})}{A(B, \hat{\theta})} \cdot f_{i}[t]+\frac{C(B, \hat{\theta})}{A(B, \hat{\theta})} \cdot e[t / \hat{\theta}]
$$

where the $n$ transfer functions $B_{i} / A \quad(i=1,2, \cdots, n)$ represent the structural dynamic characteristics, and the transfer function $C / A$ the noise dynamics. In structural dynamics identification the latter is not needed any further (although for problems where the final objective is vibration control or prediction the noise dynamics play a significant role and should be kept in the model), and is therefore discarded, whereas each one of the former $n$ transfer functions needs to be transformed back into the continuous-time domain, so that 
the parameters (natural frequency, damping factor, and mode shape) corresponding to each vibrational mode can be extracted.

This is typically done without any particular attention paid to the excitation signal form or the model structure used. The discrete-to-continuous dynamic system transformation is, however, not unique, but depends upon the intersample behavior of the excitation signals, and this information, in turn, determines some of the model structure characteristics. As a consequence, in order to achieve accurate modal parameter (mode shape) extraction, these facts need to be properly accounted for, and a transformation and model structure that are consistent with the excitation signal form need to be selected. Within the context of this work the force excitations are approximated as trains of "impulsive" functions occuring at the sampling instants, and, as it may be shown, each discrete-time transfer function is then related to its continuous-time counterpart through the transformation expression:

$$
\begin{gathered}
\frac{B_{i}(B)}{A(B)}=\sum_{k=1}^{n} \frac{\left(\Phi_{i k}+\Phi_{i k}^{*}\right)-\left(\Phi_{i k} \cdot \lambda_{k}^{*}+\Phi_{i k}^{*} \cdot \lambda_{k}\right) \cdot B}{1-\left(\lambda_{k}+\lambda_{k}^{*}\right) \cdot B+\lambda_{k} \cdot \lambda_{k}^{*} \cdot B^{2}} \Leftrightarrow \\
\Leftrightarrow \frac{B_{i}(s)}{A(s)}=\sum_{k=1}^{n} \frac{\left(\Phi_{i k}+\Phi_{i k}^{*}\right) s-\left(\Phi_{i k} \cdot \ln \lambda_{k}^{*}+\Phi_{i k}^{*} \cdot \ln \lambda_{k}\right) T^{-1}}{s^{2}+2 \zeta_{k} \cdot \omega_{n k} \cdot s+\omega_{n k}^{2}}
\end{gathered}
$$

where $\left(\Phi_{i k}, \Phi_{i k}^{*}\right)$ represent a pair of complex conjugate residues of the continuous-time transfer function, $\left(\lambda_{k}, \lambda_{k}^{*}\right)$ a pair of complex conjugate eigenvalues of the discrete transfer function, $\omega_{n k}, \zeta_{k}$, the $\mathrm{k}$-th mode natural frequency and damping factor, respectively, $s$ the Laplace Transform variable, and $T$ the selected sampling period.

From this expression it may be verified that the exogeneous polynomial order of the discrete transfer function is equal to $2 n-1$ and that no time-delay exists in the discretetime model; facts that justify the special model structure used in the modeling strategy of subsection 2.3.1. From expression (33) the $\mathrm{k}$-th mode natural frequency and damping factor 
may be evaluated as functions of the discrete eigenvalues $\left(\lambda_{k}, \lambda_{k}^{*}\right)$, and the corresponding mode shape obtained as:

$$
\psi_{k}=\left[1 \frac{\Phi_{2 k}}{\Phi_{1 k}} \cdots \frac{\Phi_{n k}}{\Phi_{1 k}}\right]^{T}
$$

In addition, the statistical dispersion percentage of the $\mathbf{k}$-th vibrational mode is evaluated for each transfer function as:

$$
\delta_{k}=\frac{d_{k}}{\sum_{i=1}^{n} d_{i}} \times 100 \%
$$

with $d_{k}$ representing the part of the vibration signal variance ("energy") due to the $k$-th mode and $\sum_{i=1}^{n} d_{i}$ the total signal variance. This quantity not only indicates the relative "strength" of each vibrational mode, but is also important as an auxiliary means of diagnosing model order overdetermination and distinguishing actual "structural" from "computational" modes.

It is finally remarked that appropriate sampling period selection is also of critical importance for accurate modal parameter extraction, since (except for the well-known sampling theorem condition) it has been shown that large errors may be introduced into the modal parameter estimates (especially in connection with multiple degree-of-freedom, lightly damped structures) when "very fast" sampling is used. This issue will not be further elaborated upon here, and the interested reader is refered to Fassois et al. (1989b) for more details.

\section{PERFORMANCE EVALUATION WITH NUMERICALLY SIMULATED VIBRATION DATA}

In this section the performance of the proposed structural dynamics identification approach is evaluated with numerically simulated vibration data. The excitation force signals used for this purpose are zero-mean and unit-variance, uncorrelated, pseudo-random Gaussian processes takning values at the sampling instances. The resulting sampled vibration signals are obtained by integrating the equations of motion by using the Wilson- $\theta$ method 
and subsequent sampling with the indicated periods. Noise-corrupted signals are generated by adding zero-mean, uncorrelated, and uncross-correlated with the excitation force signals, pseudo-random sequences to the actual vibration data. The noise-to-signal ratio is defined as the ratio of the standard deviation $\left(\sigma_{n}\right)$ of the noise over that $\left(\sigma_{v}\right)$ of the uncorrupted vibration signal:

$$
\mathrm{N} / \mathrm{S} \triangleq \frac{\sigma_{n}}{\sigma_{v}} \times 100 \%
$$

Unless otherwise indicated, data sets consisting of 1000 samples each are used. Each such set is divided into an estimation (consisting of 900 samples) and validation (consisting of 100 samples) subset, the latter being exclusively used for model validation. The discrete parameter estimation algorithm is allowed to iterate, and convergence is assumed as soon as the index:

$$
J_{i} \triangleq \frac{\left\|\hat{\theta}_{i}-\hat{\theta}_{i-1}\right\|_{I I}}{\left\|\hat{\theta}_{i-1}\right\|_{I I}} \times 100 \%
$$

where $i$ denotes the iteration number and $\|\cdot\|_{I I}$ octahedric vector norm, becomes smaller than a prespecified threshold value. The relative estimation accuracy in each iteration is then judged through the Residual Sum of Squares (RSS) :

$$
\operatorname{RSS}(i) \triangleq \sum_{t} e^{2}\left[t / \hat{\boldsymbol{\theta}}_{i}\right]
$$

where $e\left[t / \hat{\theta}_{i}\right]$ represents the one-step ahead prediction error sequence estimated at iteration $i$.

The objective of the simulation work of this section is the evaluation of the performance characteristics of the proposed approach, with special emphasis placed on issues such as:

- achievable estimation accuracy at different N/S ratios. 
- ability to distinguish closely-spaced vibrational modes (achievable resolution) at different $N / S$ ratios.

- effects of data record length on the achievable accuracy.

- effects of iterations on estimation accuracy, and convergence of the iterative procedure.

- modeling strategy effectiveness and assessment of the need for model overdetermination.

- effectiveness of the two-phase model validation and the modal parameter extraction procedures.

In addition, comparisons with the classical frequency domain method based on the Fast Fourier Transform and a Hanning window (the "direct" approach [Kay and Marple, 1981]) are made, and the inadequacy of deterministic approaches in dealing with noise-corrupted data illustrated through comparisons with the Eigensystem Realization Algorithm (ERA) (Juang and Pappa, 1985). The ERA is a time-domain, state-space, deterministic method that is based on realization theory and requires the formulation of a block Hankel matrix from the measured data. The modal parameters are computed from the minimum order realized model, whereas the effects of noise on the data are essentially attempted to be accounted for through model overdetermination and the use of accuracy indicators (such as the singular values of the block data matrix and a parameter refered to as Modal Amplitude Coherence [MAC]) that quantitatively partition the realized model into "actual" and "noise" portions, so that the latter may be neglected. It should be noted at this point that the comparisons of this section between the proposed approach and the ERA serve to illustrate the relative performance characteristics of the proposed stochastic and general deterministic methods at different $\mathrm{N} / \mathrm{S}$ ratios. The results are however only indicative, since no formal comparison 
is possible as the ERA operates on either free or impulse response data and the proposed approach on random excitation/forced response data.

Simulation results with three structural models, classified as Test Cases I, II, and III, are presented in the sequel.

\subsection{Test Case I : Two degree-of-freedom system with well-separated modes}

In this case the two degree-of-freedom system of Figure 1 with physical and corresponding modal parameters indicated in Table 1 , is identified from excitation/response data records that consist of 900 samples each. Three cases are considered: The first is an ideal nonoise case $(\mathrm{N} / \mathrm{S}=0 \%)$, whereas in the other two cases $\mathrm{N} / \mathrm{S}=10 \%$ and $32 \%$, respectively. The modeling strategy indicated ARMAX $(4,3,3,4)$ models as statistically adequate in each case, and an ARX model order of $p=12$ [see Eqns.(10)-(11)] was used in estimation. The algorithm was allowed to iterate until convergence, monitored through $\operatorname{RSS}(i)$ and the index $J_{i}$, was achieved. For the $10 \% \mathrm{~N} / \mathrm{S}$ case both of these quantities are plotted as functions of the iteration number $i$ on Figure 2, and as it may be seen, a few (in this particular case four) iterations are sufficient. The improvement that can be achieved through such additional iterations is, however, oftentimes marginal.

After the discrete adequate ARMAX model has been estimated it must be validated, and this is done by following the two-phase procedure of subsection 2.3 .2 . For the $10 \% \mathrm{~N} / \mathrm{S}$ case the model validation results are depicted in Figure 3. As it may be readily seen, the estimated normalized autocorrelation of the one-step ahead prediction error sequence associated with the model stays quite satisfactorily within the theoretical band (Figure 3a), and the onestep ahead vibration signal predictions (within the validation subset consisting of 100 data samples) are extremely close to their actual values (Figure $3 b)$. The ARMAX $(4,3,3,4)$ model 
is thus accepted as an accurate, discrete-time representation of the structural and noise dynamics.

The complete modal parameter estimation results (after modal parameter extraction has been completed) are tabulated in Table 1, where excellent accuracy is observed at all three $\mathrm{N} / \mathrm{S}$ ratios and for all types of modal parameters, including the mode shapes and damping factors that are typically hard to accurately estimate in the presence of noise. The estimated frequency response magnitude curves for the transfer function relating $x_{2}$ and $f_{1}$ (Figure 1) are compared to the theoretical curves in Figure 4, where excellent agreement is observed for all three $\mathrm{N} / \mathrm{S}$ ratio cases. In the same figure comparisons with the classical frequency domain method (FDM) are also shown, with the superior performance of the proposed approach being apparent, despite the fact that much longer data records (consisting of 5120 samples each, as opposed to 900 with the proposed method) and pseudo-ensemble averaging were used in conjunction with the FDM.

The excellent performance characteristics of the proposed approach were finally verified even with shorter $(N=200,500)$ data records, and results that indicate the achievable accuracy for the $10 \% \mathrm{~N} / \mathrm{S}$ ratio case are presented in Table 2.

\subsection{Test Case II : Two degree-of-freedom system with closely-spaced modes}

In this case the two degree-of-freedom system of Figure 1 is used once again, but its physical parameters are now selected such that the two vibrational modes are closely spaced (Table 3). The emphasis in this case thus is on the achievable resolution and the ability of the proposed approach to distinguish between the two modes, especially in the presence of noise.

Similarly to the previous case, the modeling strategy indicated ARMAX $(4,3,3,4)$ models 
as statistically adequate, and an ARX model order of $p=14$ was used in the estimation. Iterations were allowed, and the $\operatorname{RSS}(i)$ and $J_{i}$ quantities for the $10 \% \mathrm{~N} / \mathrm{S}$ ratio case are depicted in Figure 5, where convergence is achieved in the second iteration (note that the improvement from iteration 1 to 2, as judged by the RSS, is however completely insignificant). The estimated adequate models were then successfully validated, and the modal parameter estimation results for the three $(0 \%, 10 \%$, and $32 \%) \mathrm{N} / \mathrm{S}$ ratio cases are tabulated in Table 3, where excellent performance and frequency resolution characteristics are observed for all three cases! Indeed, the discrimination of the two modes is excellent even at the $32 \% \mathrm{~N} / \mathrm{S}$ ratio case, and no overfitting is needed. In the same table, modal parameter estimation results obtained by the ERA are also included, so that a feeling about the performance of deterministic methods may be gained. The ERA results were obtained by using impulse response data, specifically a $15 \times 30$ block data matrix with a $2 \times 1$ vector per block. After a few trials, a cut-off singular value of $10^{-4}$ was selected for model order selection. The Modal Amplitude Coherence (MAC) values were also computed, and a mode was considered to be true ("structural") when the corresponding MAC value was higher than $10 \%$.

As is apparent from the results of Table 3, the ERA offers excellent accuracy and resolution in the deterministic $(0 \% \mathrm{~N} / \mathrm{S})$ case, where it has no difficulty in resolving the two closelyspaced modes. This is also seen from the MAC plots of Figure 6, where only two frequencies are estimated and their MAC's equal 100\%. Nevertheless, as noise is added to the data (N/S $=10 \%, 32 \%$ ), a significantly overdetermined model is required, and the ERA identifies only one of the two modes (in fact the one with the highest dispersion percentage) while completely missing the other. As the MAC plots of Figure 6 indicate, a number of "computational" modes that are apparently due to the noise and have significant MAC values associated with them, 
are now estimated. The discrimination between true ("structural") and "computational" modes is thus, indeed, a serious problem with deterministic approaches.

\subsection{Test Case III : Three degree-of-freedom system}

In this final case the three degree-of-freedom system of Figure 7 with physical and corresponding true modal parameters given in Table 4, is identified from excitation/response data. Similarly to the previous cases, three $\mathrm{N} / \mathrm{S}$ ratios $(0 \%, 10 \%, 32 \%)$ are considered.

For the no-noise case, the modeling strategy indicated an $\operatorname{ARMAX}(6,5,5,5,6)$ model as statistically adequate, and an ARX model order of $p=20$ was selected in estimation. For the $10 \%$ and $32 \% \mathrm{~N} / \mathrm{S}$ ratio cases, however, the modeling strategy indicated that $\operatorname{ARMAX}(8,7,7,7,8)$ models are needed. This means that a slight overdetermination is necessary in this example when noise-corrupted data are used, and an ARX model order of $p=24$ was selected in the estimation. The estimation algorithm was allowed to iterate until convergence was achieved, and the models were validated before finally accepted.

The modal parameter estimation results are presented in Table 4, where comparisons with the corresponding ERA results are also shown. As is clearly seen the two methods yield essentially identical results in the no-noise case. When noise is added ( $N / S=10 \%)$ the proposed approach uses slight overdetermination and very accurately estimates all three vibrational modes (it is noted that the extra two eigenvalues estimated in both the $10 \%$ and $32 \% \mathrm{~N} / \mathrm{S}$ ratio cases are real, having completely insignificant dispersion percentages associated with them, and are therefore easy to distinguish from the true system modes). On the other hand the ERA can relatively accurately identify the first and third modes, but, despite the significant overdetermination, fails to estimate the second mode (which, not surprisingly, is the one with the smallest dispersion percentage). As more noise is added ( $\mathrm{N} / \mathrm{S}=32 \%)$, the proposed 
approach continues to provide good accuracy for all three modes, whereas the ERA now fails to estimate two (the second and third) modes. The mode estimated by the ERA is the one with the highest dispersion percentage. This example thus clearly indicates the difficulties associated with deterministic modal parameter estimation methods, on one hand, and the effectiveness of the proposed stochastic approach on the other.

Comparisons with the classical Frequency Domain Method (FDM) are also shown in Figure 8, where the theoretical and estimated (by both the proposed and FDM methods) frequency response magnitude curves for all three $\mathrm{N} / \mathrm{S}$ ratios are depicted. The superior performance of the proposed approach may be observed once again, despite the fact that much longer data records (consisting of 5120 samples each as opposed to 900 of the proposed method) and pseudo-ensemble averaging were used in conjunction with the FDM method. In Table 5 the performance characteristics of the proposed approach are finally examined with shorter $(N=200,500)$ data records for the $10 \% \mathrm{~N} / \mathrm{S}$ ratio case. As is clearly shown very good accuracy can be achieved even with records that are only 200 samples long !

\subsection{Discussion}

From the test case results of the previous subsections, as well as a number of additional simulations, the following remarks may be made :

(a) Although in the ideal noise-free case the proposed stochastic approach and available deterministic methods (such as the ERA) perform equally well in terms of achievable accuracy and resolution, the situation changes drastically as noise is added to the data. Deterministic methods may then completely fail to identify certain modes or resolve closely-spaced ones, whereas the proposed stochastic approach has no such difficulties and accurately estimates all vibrational modes, including closely-spaced ones, at all $\mathrm{N} / \mathrm{S}$ ratios considered. 
(b) When compared to the classical frequency domain method (FDM), the proposed approach offers superior performance even with significantly shorter data records. The need for subjective judgement, pseudo-ensemble averaging, window selection (and its effects on estimation accuracy), subsequent curve-fitting, along with the other well-known drawbacks of frequency domain methods, are thus eliminated.

(c) The proposed stochastic approach is capable of offering high estimation accuracy from noise-corrupted and short data records (in the test cases considered, data records consisting of as few as 200 samples were used).

(d) In achieving the best possible accuracy the discrete estimation algorithm may be allowed to iterate a few times, until convergence is achieved. This typically happens in the first two to three iterations in the noise-free case, while a few more may be required when noise-corrupted data are used. Nevertheless, the improvement offered by the additional iterations is typically small, and in certain cases may be even marginal.

(e) The modeling strategy of the proposed approach appears to be very effective in selecting the model orders required for accurate modal parameter estimation. Unlike deterministic techniques that heavily rely on excessive model order overdetermination and the inherently difficult procedure of distinguishing "computational" from actual "structural" modes, the proposed approach may need either slight or no overdetermination at all. Furthermore, in those cases where overdetermination is required, this is clearly indicated by the modeling strategy, and the additional ("false") estimated modes are identified as such via the dispersion analysis, since their associated dispersion percentages have been observed to be completely insignificant. 
(f) The two-phase model validation procedure has been found to be very effective in screening the estimated structural and noise dynamics models and indicating any potential deficiencies.

(g) The modal parameter extraction is finally sufficiently accurate, provided that the excitation signal can be reasonably well approximated by a train of "impulsive" functions of various strengths, the sampling period is appropriately selected, and the correct model structure used.

\section{EXPERIMENTAL RESULTS : MODAL ANALYSIS OF A FLEXIBLE BEAM}

In this section the proposed structural dynamics identification approach is used for the modal analysis of a flexible beam from laboratory data. The frequency range of interest was selected as 0-120 Hz, and linear approximate analysis indicated the existence of three vibrational modes, with frequencies listed in Table 6, in that range. The damping factors cannot be analytically predicted, but are expected to be small.

The schematic diagram of the experimental set-up is shown in Figure 9. The beam was excited by band-limited zero-mean and uncorrelated force signals applied through an electromagnetic exciter and measured by a load cell. Since the beam is lightweight, the vibration displacement signals were measured through a non-contacting device (proximity probe). The force excitation and corresponding response signal pairs were subsequently amplified, low-pass filtered with a cut-off frequency of $140 \mathrm{~Hz}$, and sampled at a sampling frequency of $500 \mathrm{~Hz}$ by using a data acquisition system consisting of an IBM AT computer equipped with the DT2821 data acquisition board.

Modeling results are presented for one such pair of excitation force and resulting vibration signals that are depicted in Figure 10. Each set consists of 1000 samples, 900 of which 
were used for estimation and the remaining 100 for model validation. The modeling strategy indicated an $\operatorname{ARMAX}(6,5,6)$ model as statistically adequate, and an ARX model order of $p=15$ was used in estimation. The algorithm was allowed to iterate, and, as Figure 11 indicates, convergence was achieved in the sixth iteration. The estimated model was subsequently successfully validated by using the two-phase procedure of subsection 2.3.2. As the results of Figure 12 indicate, the estimated normalized autocorrelation of the one-step ahead prediction error sequence stays within the theoretical band, and the one-step ahead vibration signal predictions are extremely close to their actual values.

The modal parameter estimation results are finally shown in Table 6, where excellent agreement with the approximate, analytically predicted natural frequency values is observed. The estimated damping factors are also very small, and well within their expected range of values for this lightly-damped system. The estimated frequency response magnitude curve is finally depicted in Figure 13.

\section{CONCLUDING REMARKS}

In this paper a highly-effective, stochastic, and multiple excitation structural dynamics identification approach, that overcomes the well-known difficulties of the currently available techniques, was introduced. The proposed approach employs special forms of discrete ARMAX representations that explicitly account for both the structural and noise dynamics, and is based on a novel Suboptimum Maximum Likelihood (SML) discrete estimation algorithm, efficient modeling strategy and model validation procedures, as well as appropriate modal parameter extraction.

The performance of the approach was evaluated with structural models characterized by both well-separated and closely-spaced modes and data corrupted at different noise levels. 
For this purpose both simulated and actual experimental vibration data were used, and comparisons with a deterministic approach (ERA) and the classical frequency domain method (FDM), made. As the results indicated, the proposed approach effectively circumvents the difficulties relating to the inadequacy of deterministic techniques in dealing with noisy data, and offers high accuracy and resolution even at relatively high noise-to-signal (N/S) ratios while effectively overcoming the severe drawbacks of alternative stochastic methods.

The unique features and advantages of the proposed approach may be thus summarized as follows:

1. High accuracy and resolution even with short and noise-corrupted data records.

2. Complete elimination of well-known drawbacks, such as the occurrence of algorithmic instabilities, local minima/convergence to an erroneous model, and excessive computational complexity, that are associated with alternative stochastic methods. Indeed, the proposed approach offers mathematically guaranteed algorithmic stability, a unique estimated model that is mathematically shown to converge to the true structural model (consistency), and significantly reduced computational complexity.

3. Minimal operator intervention and absolutely no need for initial guess parameter values.

4. Very effective modeling strategy and model validation procedures. Excessive overdetermination that is typically required with deterministic techniques is effectively circumvented.

5. Accurate modal parameter extraction that is consistent with the employed model structure and excitation signal forms. 
6. Ability to perform modal analysis based on multiple-excitation data; a feature known to significantly contribute in achieving high quality estimation while significantly reducing the data acquisition and processing time requirements. 


\section{ACKNOWLEDGEMENT}

Financial support for this work through a Rackham Faculty Grant for Research at the University of Michigan is gratefully acknowledged. 


\section{REFERENCES}

Akaike, H., 1974, "A New Look at the Statistical Model Identification", IEEE Transactions on Automatic Control, Vol. 19, pp. 716-723.

Åström, K. J., 1970, "Introduction to Stochastic Control Theory," Academic Press.

Åström, K. J., 1980, "Maximum Likelihood and Prediction Error Methods", Automatica, Vol. 16, pp. 551-574.

Ben Mrad, R., and Fassois, S.D., 1989, "Recursive Identification of Vibrating Structures from Noise-Corrupted Observations", Proceedings of the ASME Winter Annual Meeting, San Francisco, CA.

Braun, S.G., and Ram, Y.M., 1987, "Time and Frequency Identification Methods in Over-Determined Systems", Mechanical Systems and Signal Processing, Vol. 1, pp. 245-257.

Davies, P., and Hammond, J. K., 1984, "A Comparison of Fourier and Parametric Methods for Structural System Identification," ASME Journal of Vibration, Acoustics, Stress, and Reliability in Design, Vol. 106, pp. 40-48.

Fassois, S. D., and Lee, J. E., 1988, "Suboptimum Maximum Likelihood Identification of ARMAX Processes," ASME Paper 88-WA/DSC-32. Also to appear in the ASME Journal of Dynamic Systems, Measurement, and Control.

Fassois, S. D., Eman, K. F., and Wu, S. M., 1989a, "A Linear Time-Domain Method for Structural Dynamics Identification," ASME Journal of Vibration, Acoustics, Stress, and Reliability in Design, in press.

Fassois, S. D., Eman, K. F., and Wu, S. M., 1989b, "Sensitivity Analysis of the DiscreteTo-Continuous Dynamic System Transformation", ASME Journal of Dynamic Systems, Measurement, and Control, in press.

Fritzen, C.P., 1986, "Identification of Mass, Damping, and Stiffness Matrices of Mechanical Systems", ASME Journal of Vibration, Acoustics, Stress, and Reliability in Design, Vol. 108, pp. 9-16.

Ibrahim, S.R., and Mikulcik, E.C., 1973, "A Time Domain Vibration Test Technique," Shock and Vibration Bulletin, No. 43, Part 4.

Juang, J.N., and Pappa, R.S., 1985, "An Eigensystem Realization Algorithm for Modal Parameter Identification and Model Reduction", Journal of Guidance, Control, and Dynam- 
ics, Vol. 8, pp. 620-627.

Juang, J.N., and Pappa, R.S., 1986, "Effects of Noise on Modal Parameters Identified by the Eigensystem Realization Algorithm", Journal of Guidance, Control, and Dynamics, Vol. 9, No. 3, pp. 294-303.

Kay, S. M., and Marple, S. L., 1981, "Spectrum Analysis - A Modern Perspective," Proceedings of IEEE, Vol. 69, No. 11, pp. 1380-1419.

Kim, K.J., Eman, K.F., and Wu, S.M., 1984, "Identification of Machine Tool Structures by the Dynamic Data System Approach", Int. J. Mach. Tool Des. Res., Vol. 24, No. 3, pp. 161-169.

Lee, G. M., and Trethewey, M. W., 1988, "Application of the Least-Squares Time Domain Algorithm for Efficient Modal Parameter Estimation," Mechanical Systems and Signal Processing, Vol. 2, pp. 21-38.

Leuridan, J.M., Brown, D.L., and Allemang, R.J., 1986, "Time Domain Parameter Identification Methods for Linear Modal Analysis: A Unifying Approach", ASME Journal of Vibration, Acoustics, Stress, and Reliability in Design, Vol. 108, pp.1-8.

Pandit, S. M., and Wu, S. M., 1983, "Time Series and System Analysis with Applications," John Wiley and Sons.

Snoeys, R., Sas, P., Heylen, W., and Van Der Auweraer, H., 1987, "Trends in Experimental Modal Analysis", Mechanical Systems and Signal Processing, Vol. 1, pp. 5-27.

Söderström, T., 1975, "On the Uniqueness of Maximum Likelihood Identification," $A u$ tomatica, Vol. 11, pp. 193-197.

Vold, H., and Rocklin, T., 1982, "The Numerical Implementation of a Multi-Input Modal Estimation Method for Mini-Computers", Proc. of the 1st International Modal Analysis Conference, Orlando, Fl, pp. 542-548. 


\begin{tabular}{|c|c|c|c|c|}
\hline & \multirow{2}{*}{$\begin{array}{l}\text { Theoretical } \\
\text { Parameters }\end{array}$} & \multicolumn{3}{|c|}{ Estimated Parameters } \\
\hline & & $\mathrm{N} / \mathrm{S}=0 \%$ & $\mathrm{~N} / \mathrm{S}=10 \%$ & $\mathrm{~N} / \mathrm{S}=32 \%$ \\
\hline Natural & 0.1090 & 0.1090 & 0.1090 & 0.1089 \\
\hline Frequencies $(\mathrm{Hz})$ & 0.4647 & 0.4644 & 0.4638 & 0.4654 \\
\hline Damping & 0.0651 & 0.0652 & 0.0656 & 0.0705 \\
\hline Factors & 0.0875 & 0.0873 & 0.0889 & 0.0855 \\
\hline \multirow{3}{*}{$\begin{array}{l}\text { Mode } \\
\text { Shapes }\end{array}$} & {$\left[\begin{array}{c}1.0 \\
1.1323+ \\
j 0.0144\end{array}\right]$} & {$\left[\begin{array}{c}1.0 \\
1.1322+ \\
j 0.0145\end{array}\right]$} & {$\left[\begin{array}{c}1.0 \\
1.1328+ \\
j 0.0141\end{array}\right]$} & {$\left[\begin{array}{c}1.0 \\
1.1357+ \\
j 0.0064\end{array}\right]$} \\
\hline & {$[1.0]$} & {$[1.0]$} & $=1.07$ & 1.0 \\
\hline & {$\left[\begin{array}{c}-0.8785+ \\
j 0.0478\end{array}\right]$} & {$\left[\begin{array}{c}-0.8786+ \\
j 0.0477\end{array}\right]$} & {$\left[\begin{array}{c}-0.8679+ \\
j 0.0446\end{array}\right]$} & {$\left[\begin{array}{c}-0.7848+ \\
j 0.0636\end{array}\right]$} \\
\hline Dispersion & 98.15 & 98.38 & 99.14 & 99.15 \\
\hline $\begin{array}{l}\text { Percentages (\%) } \\
\left(\mathrm{TF} x_{2} / f_{1}\right)\end{array}$ & 1.85 & 1.62 & 0.86 & 0.85 \\
\hline \multicolumn{5}{|c|}{$\begin{array}{l}\text { Sampling Interval : } 0.538 \mathrm{sec} \\
\text { System : Figure } 1 \\
m_{1}=1 \mathrm{~kg}, m_{2}=1 \mathrm{~kg} \\
c_{1}=0.2 \mathrm{~N} \cdot \mathrm{s} / \mathrm{m}, c_{2}=0.2 \mathrm{~N} \cdot \mathrm{s} / \mathrm{m}, c_{3}=0 \mathrm{~N} \cdot \mathrm{s} / \mathrm{m} \\
k_{1}=1 \mathrm{~N} / \mathrm{m}, k_{2}=4 \mathrm{~N} / \mathrm{m}, k_{3}=0 \mathrm{~N} / \mathrm{m}\end{array}$} \\
\hline
\end{tabular}

Table 1. Modal parameters estimated by the proposed approach at different $\mathrm{N} / \mathrm{S}$ ratios (Test Case I).

\begin{tabular}{|r|c|c|c|} 
& $\begin{array}{c}\text { Natural } \\
\text { Frequencies } \\
(\mathrm{Hz})\end{array}$ & $\begin{array}{c}\text { Damping } \\
\text { Factors }\end{array}$ & $\begin{array}{c}\text { Dispersion } \\
\text { Percentages } \\
(\%)\end{array}$ \\
\hline Theoretical & 0.1090 & 0.0651 & 98.15 \\
Parameters & 0.4647 & 0.0875 & 1.85 \\
\hline No. of Data : & 0.1091 & 0.0659 & 99.35 \\
200 & 0.4665 & 0.1005 & 0.65 \\
\hline No. of Data : & 0.1090 & 0.0654 & 99.21 \\
500 & 0.4646 & 0.0971 & 0.79 \\
\hline No. of Data : & 0.1090 & 0.0656 & 99.14 \\
900 & 0.4638 & 0.0889 & 0.86 \\
\hline
\end{tabular}

Table 2. Modal parameters estimated by the proposed approach from data sets of various lengths (Test Case I; N/S = $10 \%$ ). 


\begin{tabular}{|l|c|c|c|c|c|c|c|}
\hline & \multirow{2}{*}{ Theoretical } & \multicolumn{6}{|c|}{ Estimated Parameters } \\
\cline { 3 - 8 } & & \multicolumn{2}{|c|}{$\mathrm{N} / \mathrm{S}=0 \%$} & \multicolumn{2}{|c|}{ N/S $=10 \%$} & \multicolumn{2}{c|}{ N/S =32\% } \\
\cline { 3 - 8 } & Parameters & SML & ERA & SML & ERA & SML & ERA \\
\hline Natural & 9.9793 & 9.9742 & 9.9728 & 9.9579 & - & 9.9662 & - \\
Frequencies (Hz) & 9.9274 & 9.9203 & 9.9209 & 9.9218 & 9.9196 & 9.9108 & 9.9100 \\
\hline Damping & 0.1826 & 0.1823 & 0.1825 & 0.1819 & - & 0.2082 & - \\
Factors & 0.0480 & 0.0479 & 0.0479 & 0.0477 & 0.0437 & 0.0455 & 0.0436 \\
\hline Sampling Interval : $0.025 \mathrm{sec}$ \\
System : Figure 1 \\
$m_{1}=4.5 \mathrm{~kg}, m_{2}=4.5 \mathrm{~kg}$ \\
$c_{1}=45 \mathrm{~N} \cdot \mathrm{s} / \mathrm{m}, c_{2}=35 \mathrm{~N} \cdot \mathrm{s} / \mathrm{m}, c_{3}=15 \mathrm{~N} \cdot \mathrm{s} / \mathrm{m}$ \\
$k_{1}=17500 \mathrm{~N} / \mathrm{m}, k_{2}=100 \mathrm{~N} / \mathrm{m}, k_{3}=17500 \mathrm{~N} / \mathrm{m}$ \\
\hline
\end{tabular}

Table 3. Modal parameters of the system with closely-spaced modes (Test Case II) estimated by the proposed (SML) and ERA methods at different N/S ratios.

\begin{tabular}{|c|c|c|c|c|c|c|c|}
\hline & \multirow{3}{*}{$\begin{array}{l}\text { Theoretical } \\
\text { Parameters }\end{array}$} & \multicolumn{6}{|c|}{ Estimated Parameters } \\
\hline & & \multicolumn{2}{|c|}{$\mathrm{N} / \mathrm{S}=0 \%$} & \multicolumn{2}{|c|}{$\mathrm{N} / \mathrm{S}=10 \%$} & \multicolumn{2}{|c|}{$\mathrm{N} / \mathrm{S}=32 \%$} \\
\hline & & SML & ERA & SML & ERA & SML & ERA \\
\hline Natural & 1.2926 & 1.2928 & 1.2925 & 1.2930 & 1.2852 & 1.2891 & 1.2901 \\
\hline \multirow{2}{*}{ Frequencies $(\mathrm{Hz})$} & 2.0622 & 2.0612 & 2.0614 & 2.0846 & $\overline{-}$ & 2.2104 & 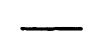 \\
\hline & 2.8295 & 2.8276 & 2.8277 & 2.8345 & 2.8786 & 2.9121 & - \\
\hline$\overline{\text { Damping }}$ & 0.0464 & 0.0465 & 0.0465 & 0.0461 & 0.0480 & 0.0513 & 0.0478 \\
\hline \multirow[t]{2}{*}{ Factors } & 0.0683 & 0.0684 & 0.0684 & 0.0894 & $\longrightarrow$ & 0.0856 & 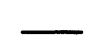 \\
\hline & 0.0612 & 0.0610 & 0.0610 & 0.0630 & 0.0770 & 0.0739 & - \\
\hline Dispersion & 87.78 & 87.77 & & 87.60 & & 88.33 & \\
\hline Percentages (\%) & 1.70 & 1.70 & & 2.52 & & 4.35 & - \\
\hline$\left(\mathrm{TF} x_{2} / f_{1}\right)$ & 10.52 & 10.53 & & 9.88 & & 7.32 & \\
\hline \multicolumn{8}{|c|}{$\begin{array}{l}\text { Sampling Interval : } 0.0884 \mathrm{sec} \\
\text { System : Figure } 7 \\
m_{1}=1 \mathrm{~kg}, m_{2}=1 \mathrm{~kg}, m_{3}=2 \mathrm{~kg} \\
c_{1}=0.6 \mathrm{~N} \cdot \mathrm{s} / \mathrm{m}, c_{2}=0.5 \mathrm{~N} \cdot \mathrm{s} / \mathrm{m}, c_{3}=0.6 \mathrm{~N} \cdot \mathrm{s} / \mathrm{m} \\
c_{4}=1.5 \mathrm{~N} \cdot \mathrm{s} / \mathrm{m}, c_{5}=0.5 \mathrm{~N} \cdot \mathrm{s} / \mathrm{m}, c_{6}=0.7 \mathrm{~N} \cdot \mathrm{s} / \mathrm{m} \\
k_{1}=100 \mathrm{~N} / \mathrm{m}, k_{2}=100 \mathrm{~N} / \mathrm{m}, k_{3}=100 \mathrm{~N} / \mathrm{m}, k_{4}=200 \mathrm{~N} / \mathrm{m}\end{array}$} \\
\hline
\end{tabular}

Table 4. Modal parameters estimated by the proposed (SML) and ERA methods at different $\mathrm{N} / \mathrm{S}$ ratios (Test Case III). 


\begin{tabular}{|r|c|c|c|}
\hline & $\begin{array}{c}\text { Natural } \\
\text { Frequencies } \\
(\mathrm{Hz})\end{array}$ & $\begin{array}{c}\text { Damping } \\
\text { Factors }\end{array}$ & $\begin{array}{c}\text { Dispersion } \\
\text { Percentages } \\
(\%)\end{array}$ \\
\hline Theoretical & 1.2926 & 0.0464 & 87.78 \\
Parameters & 2.0622 & 0.0683 & 1.70 \\
& 2.8295 & 0.0612 & 10.52 \\
\hline No. of Data: & 1.2973 & 0.0454 & 85.40 \\
200 & 2.1146 & 0.1157 & 2.36 \\
& 2.8435 & 0.0728 & 12.24 \\
\hline No. of Data: & 1.2922 & 0.0468 & 88.41 \\
500 & 2.0706 & 0.0670 & 1.80 \\
& 2.8351 & 0.0635 & 9.79 \\
\hline No. of Data: & 1.2930 & 0.0461 & 87.65 \\
900 & 2.0846 & 0.0894 & 2.20 \\
& 2.8345 & 0.0630 & 10.15 \\
\hline
\end{tabular}

Table 5. Modal parameters estimated by the proposed approach from data sets of various lengths (Test Case III ; N/S $=10 \%$ ).

\begin{tabular}{|c|c|c|c|}
\hline $\begin{array}{c}\text { Theoretical } \\
\text { Natural } \\
\text { Frequencies } \\
(\mathrm{Hz})\end{array}$ & $\begin{array}{c}|c| \\
\text { Estimated Parameters } \\
\text { Frequencies } \\
(\mathrm{Hz})\end{array}$ & $\begin{array}{c}\text { Damping } \\
\text { Factors }\end{array}$ & $\begin{array}{c}\text { Dispersion } \\
\text { Percentages } \\
(\%)\end{array}$ \\
\hline 19.81 & 19.3998 & 0.0041 & 105.50 \\
56.41 & 55.3835 & 0.0243 & -5.48 \\
108.71 & 107.2029 & 0.0056 & -0.02 \\
\hline
\end{tabular}

Table 6. Modal parameters of the beam as estimated by the proposed approach. 


\section{LIST OF FIGURES}

Figure 1: The two degree-of-freedom vibratory system used in Test Cases I and II.

Figure $2:$ The RSS and $J_{i}$ as functions of the iteration number (Test Case I; N/S $=10 \%$ ).

Figure 3 : Validation of the estimated model: (a) The normalized autocorrelation of the residuals (b) One-step ahead predictions of the vibration signal (Test Case I; N/S = 10\%).

Figure $4:$ The frequency response magnitude curves as estimated by the proposed (SML) and classical frequency domain (FDM) approaches at different $\mathrm{N} / \mathrm{S}$ ratios (Test Case I).

Figure 5 : The RSS and $J_{i}$ as functions of the iteration number (Test Case II; N/S = $10 \%)$.

Figure 6 : The Modal Amplitude Coherence (MAC) of the frequencies estimated by the ERA method at different $\mathrm{N} / \mathrm{S}$ ratios (Test Case II).

Figure $7:$ The three degree-of-freedom vibratory system used in Test Case III.

Figure $8:$ The frequency response magnitude curves as estimated by the proposed (SML) and classical frequency domain (FDM) approaches at different $\mathrm{N} / \mathrm{S}$ ratios (Test Case III).

Figure 9 : Schematic diagram of the experimental set-up.

Figure 10 : The experimental data: (a) Excitation (b) Response.

Figure 11 : The RSS and $J_{i}$ as functions of the iteration number (Beam Experiment).

Figure 12 : Validation of the estimated model: (a) The normalized autocorrelation of the residuals (b) One-step ahead predictions of the vibration signal (Beam Experiment).

Figure 13 : The frequency response magnitude curve as estimated by the proposed approach (Beam Experiment). 


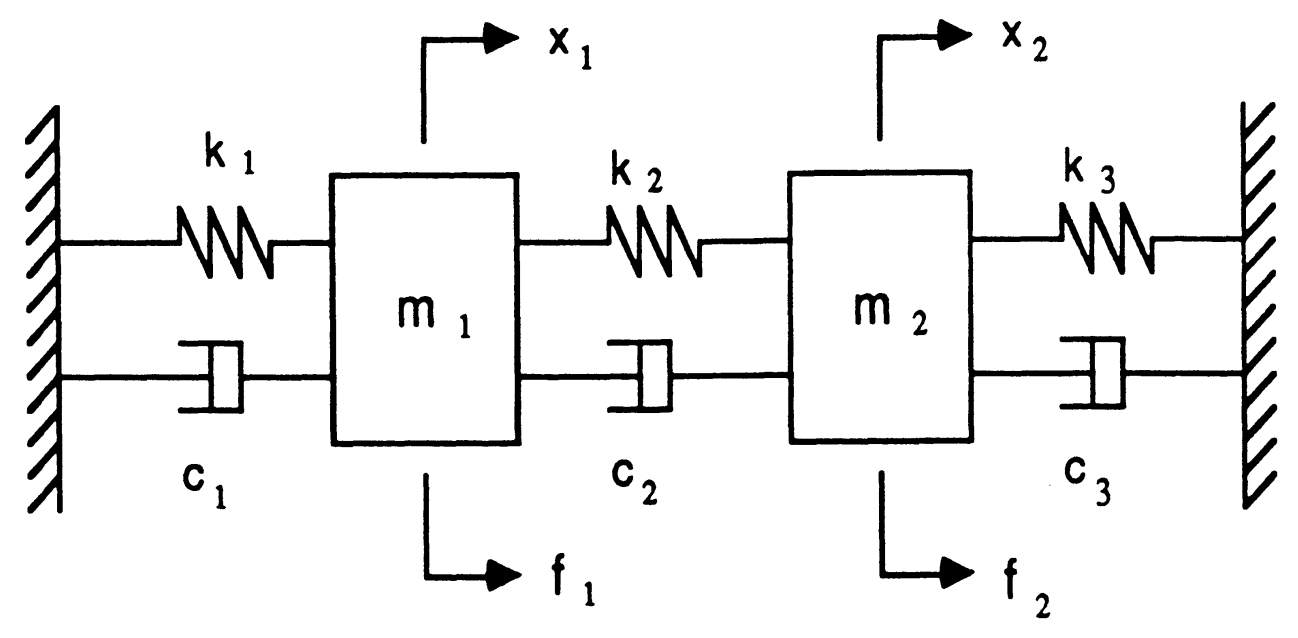

Figure 1: The two degree-of-freedom vibratory system used in Test Cases I and II. 

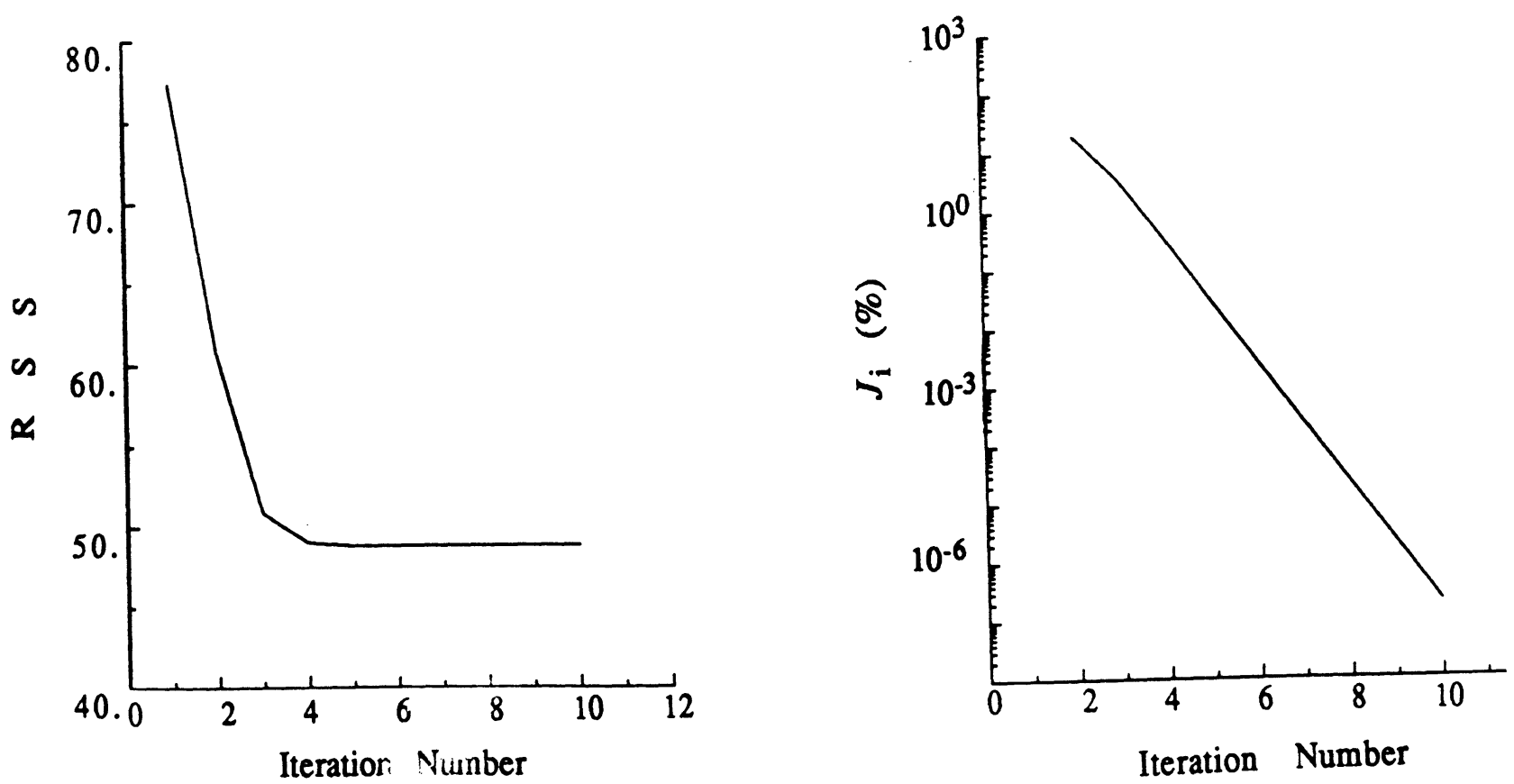

Figure 2 : The RSS and $J_{i}$ as functions of the iteration number (Test Case I; N/S $=10 \%$ ).

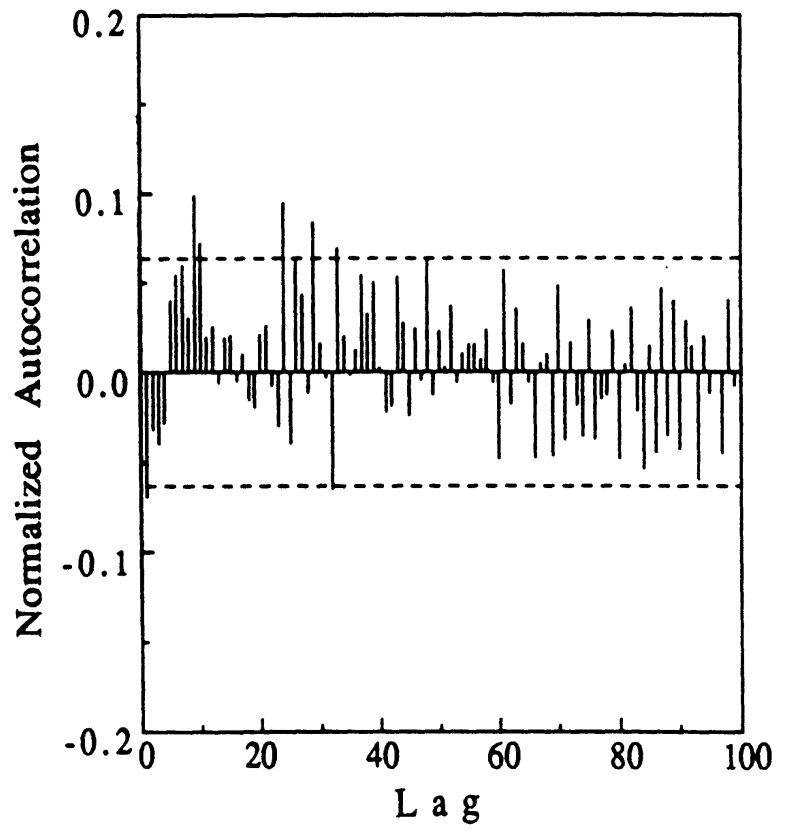

(a)

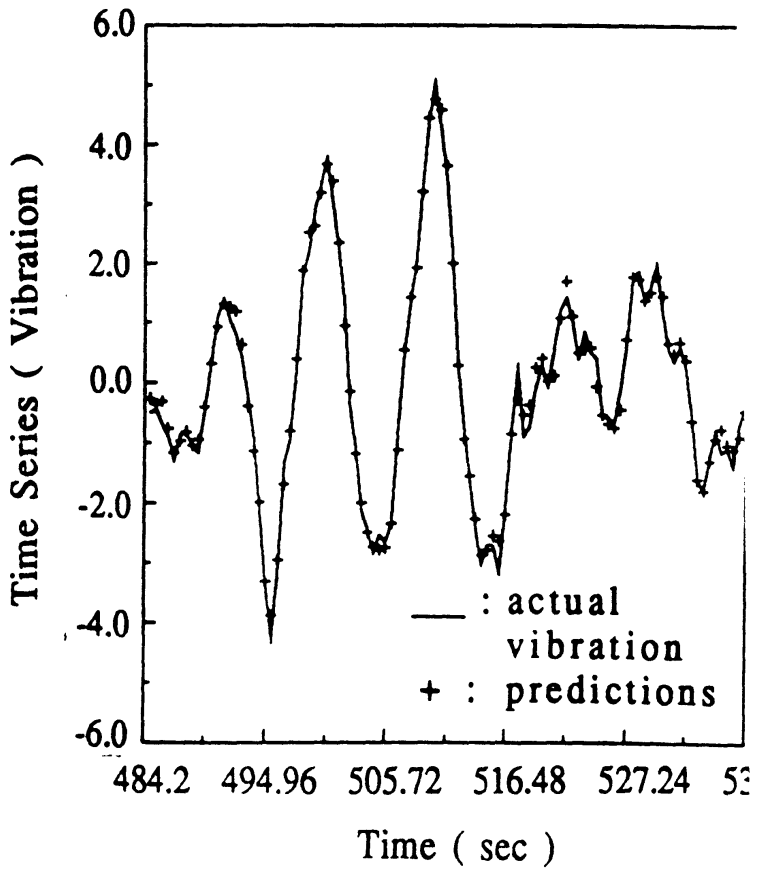

(b)

Figure 3 : Validation of the estimated model: (a) The normalized autocorrelation of the residuals (b) One-step ahead predictions of the vibration signal (Test Case I; N/S = 10\%). 

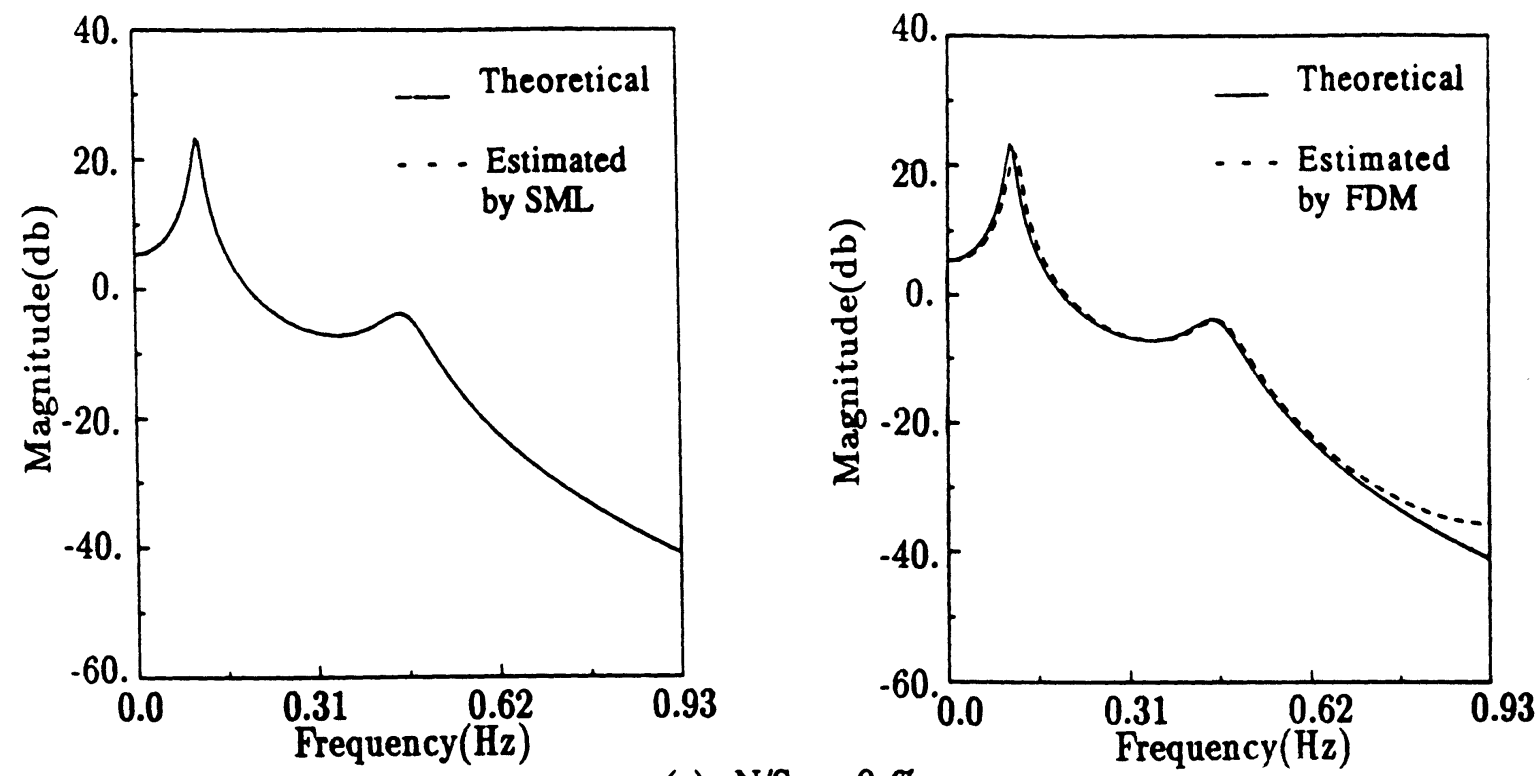

(a) $\mathrm{N} / \mathrm{S}=0 \%$
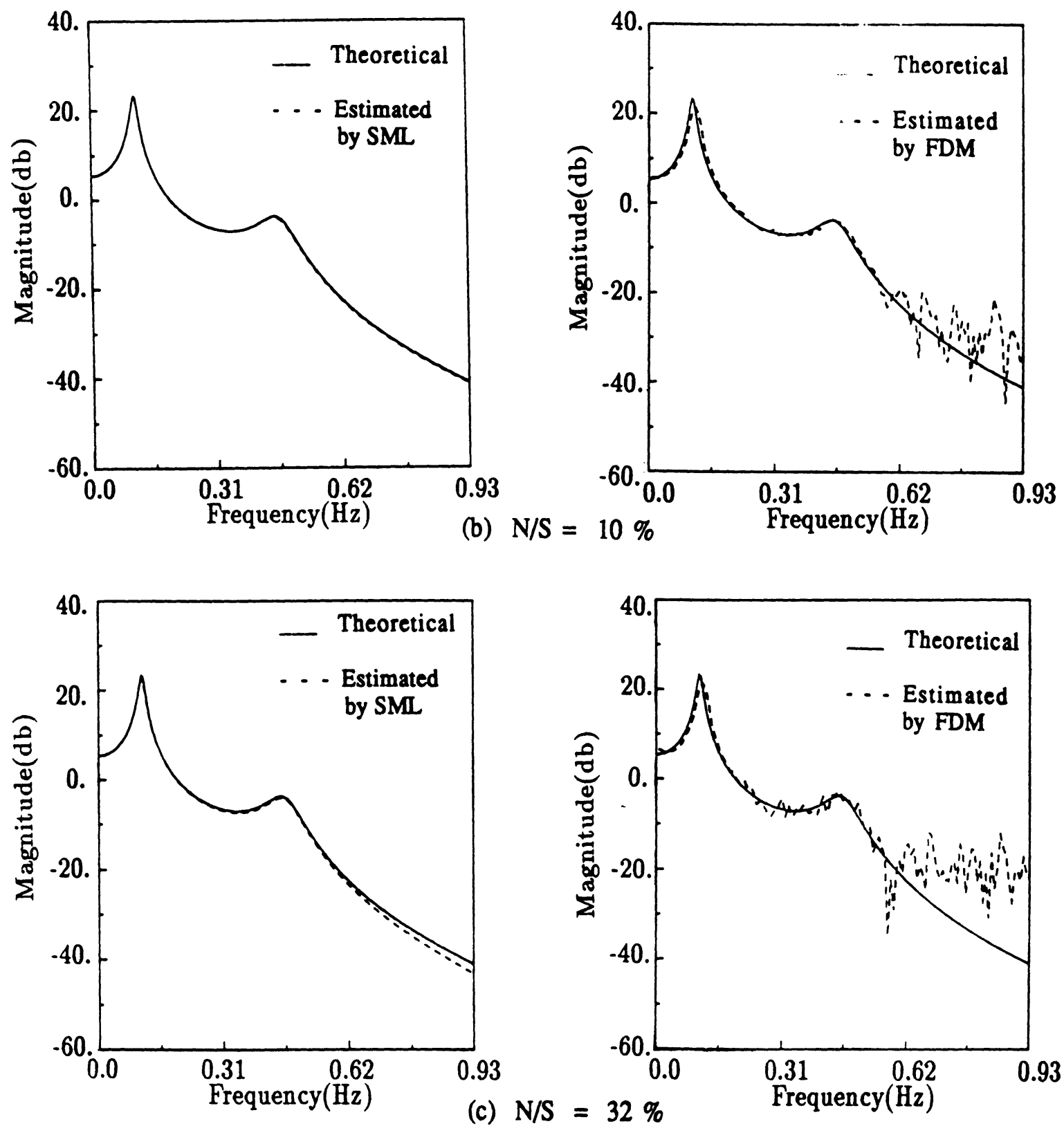

Figure $4:$ The frequency response magnitude curves as estimated by the proposed (SML) and classical frequency domain (FDM) approaches at different $N / S$ ratios (Test Case I). 

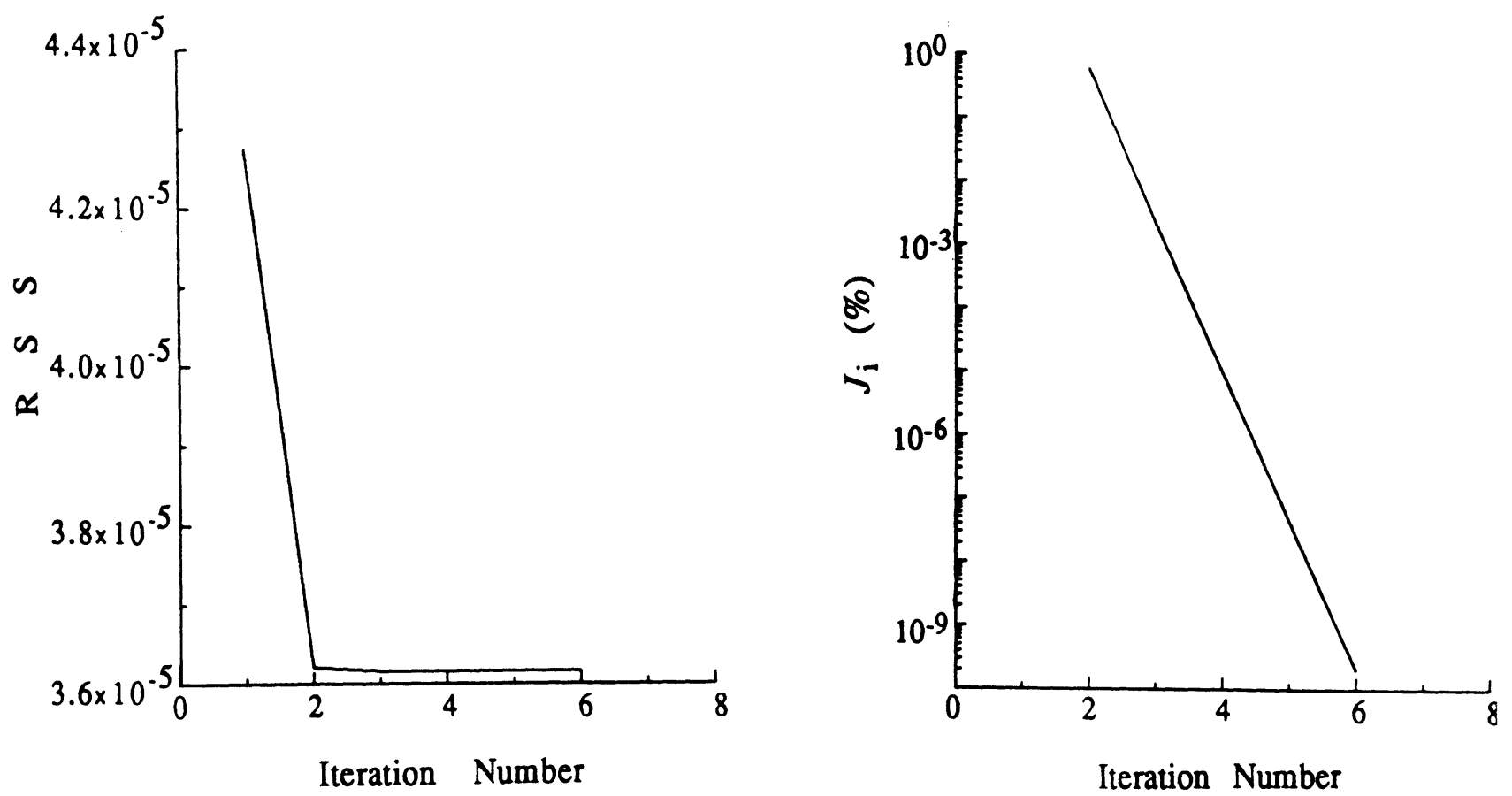

Figure 5 : The RSS and $J_{i}$ as functions of the iteration number (Test Case II; N/S $=10 \%$ ). 


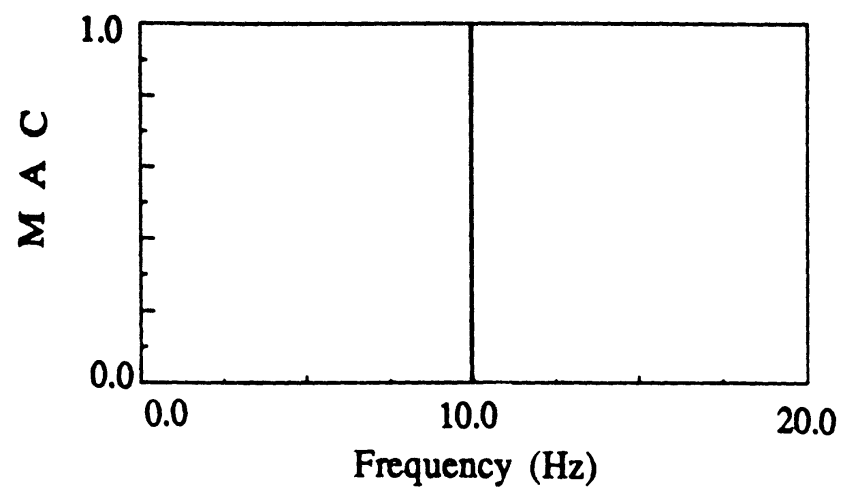

(a) Theoretical

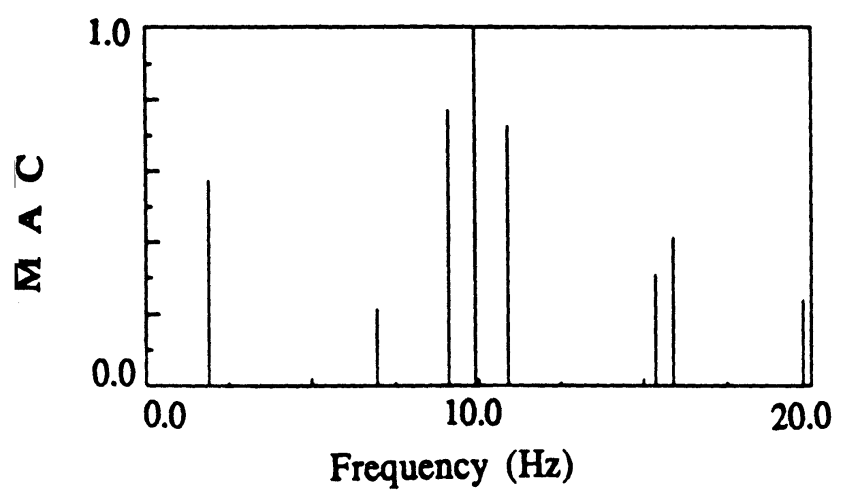

(c) Estimated ( $\mathrm{N} / \mathrm{S}=10 \%)$

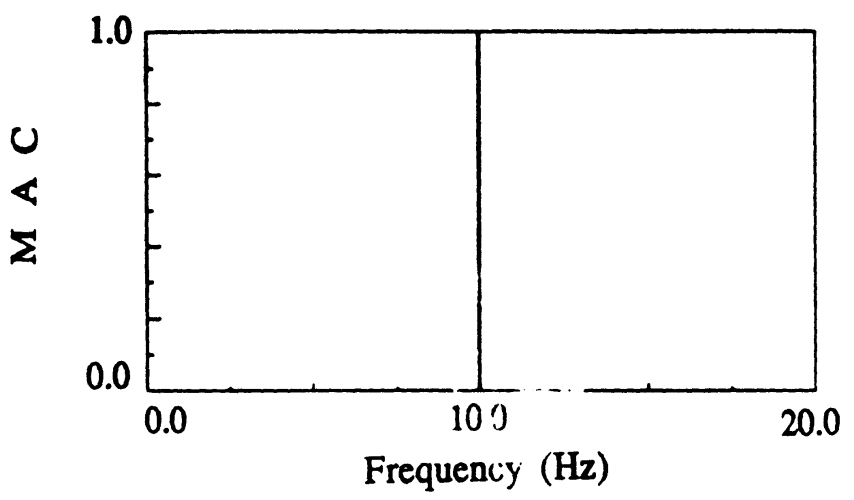

(b) Estimated ( N/S = $0 \%)$

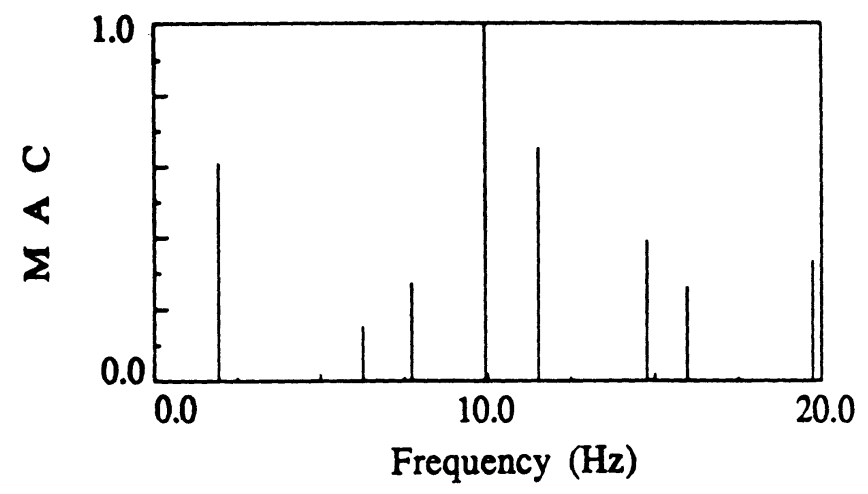

(d) Estimated ( N/S = $32 \%$ )

Figure 6 : The Modal Amplitude Coherence (MAC) of the frequencies estimated by the ERA method at different $\mathrm{N} / \mathrm{S}$ ratios (Test Case II). 


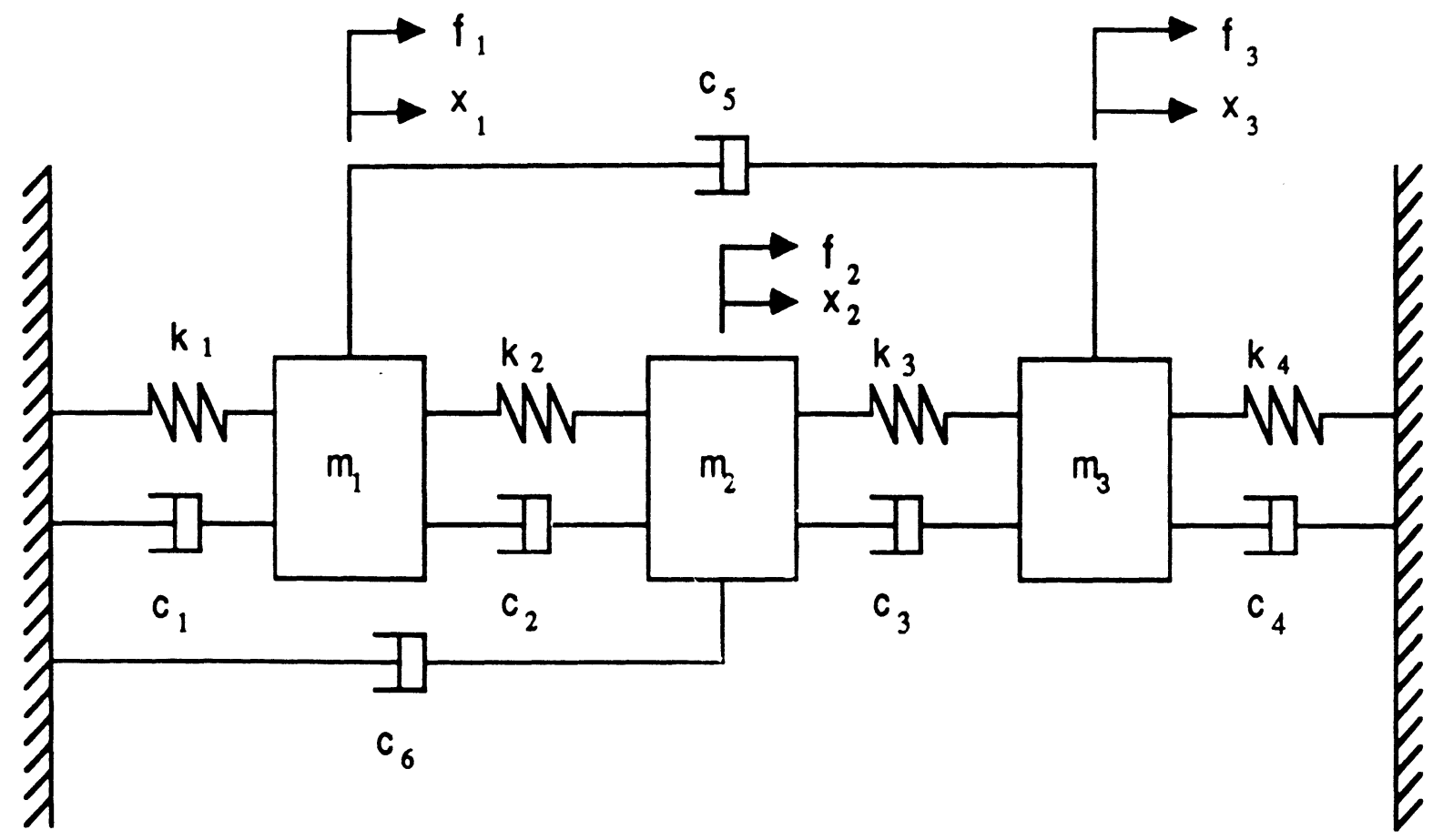

Figure 7 : The three degree-of-freedom vibratory system used in Test Case III. 

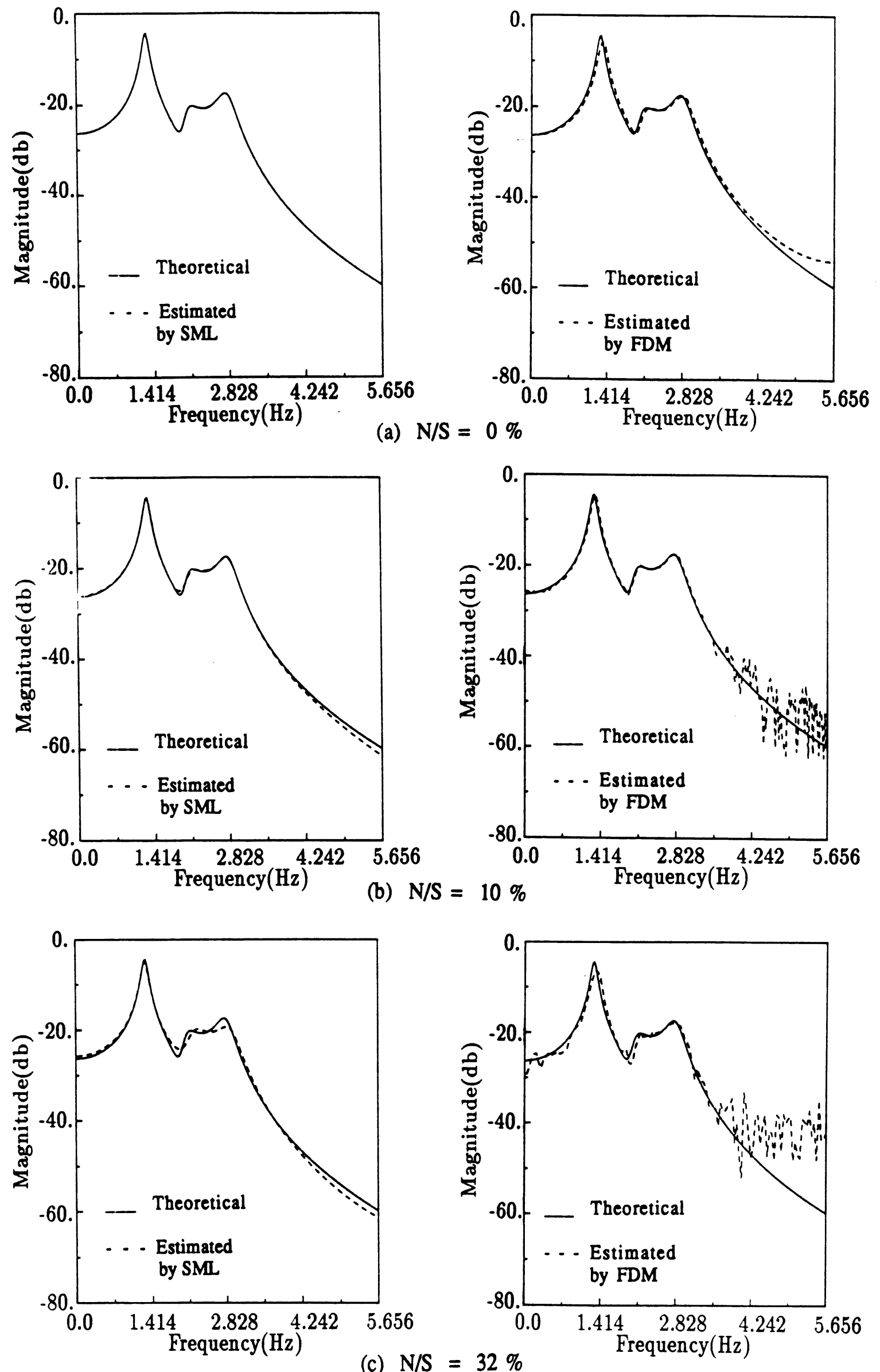

Figure 8 : The frequency response magnitude curves as estimated by the proposed (SML) and classical frequency domain (FDM) approaches at different $\mathrm{N} / \mathrm{S}$ ratios (Test Case III). 


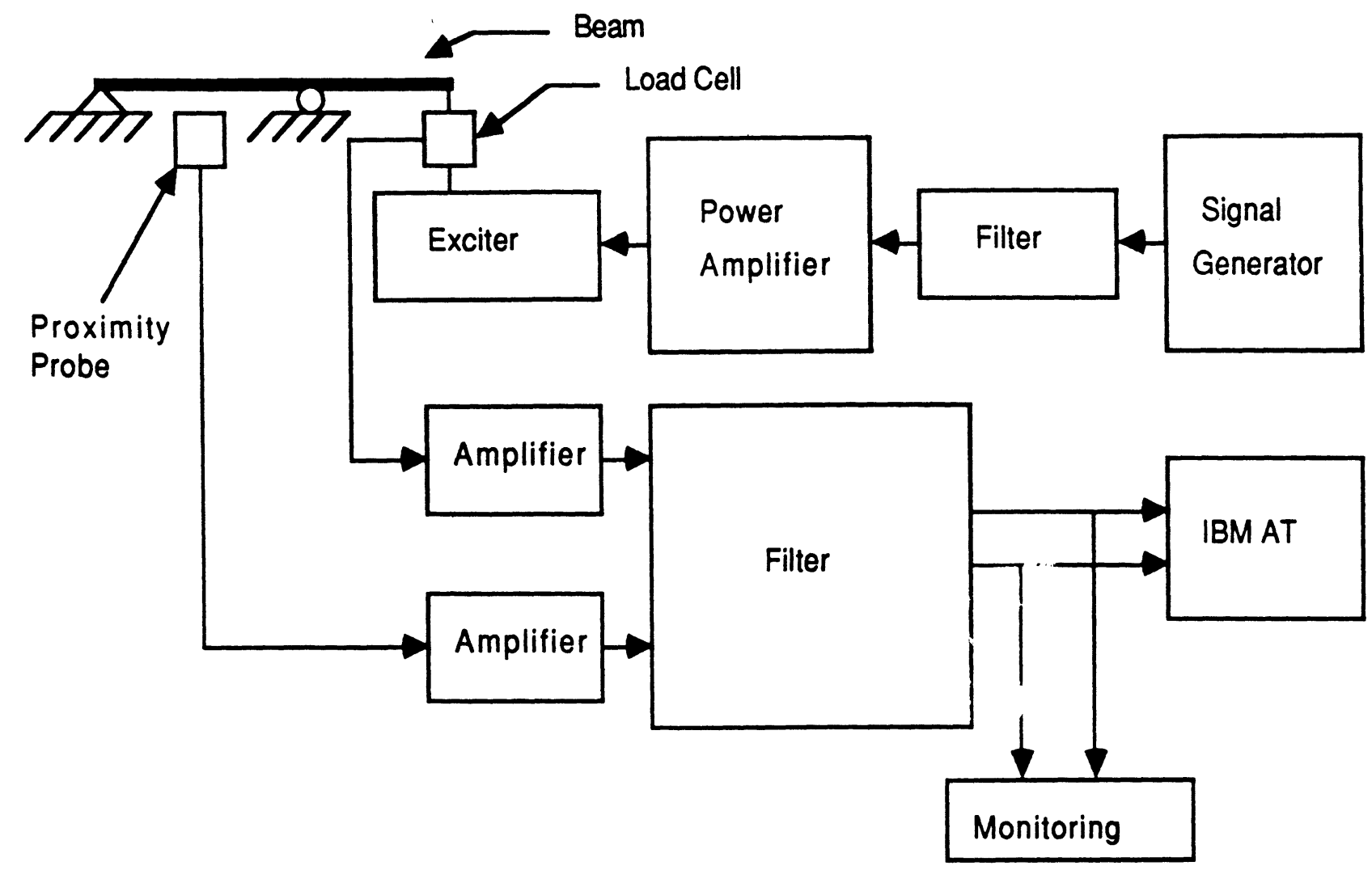

Figure 9 : Schematic diagram of the experimental set-up. 


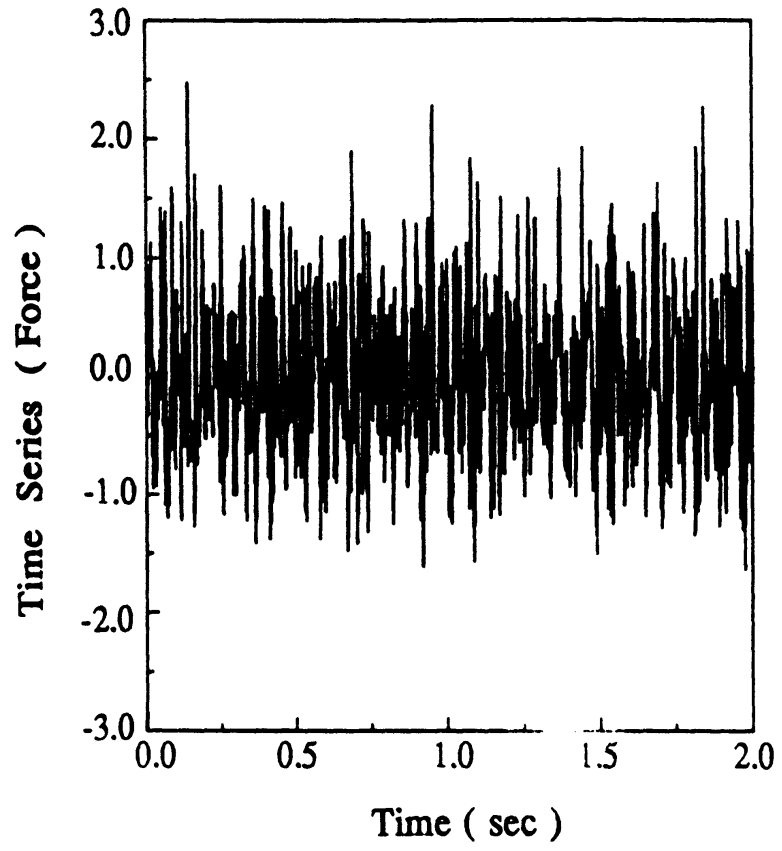

(a)

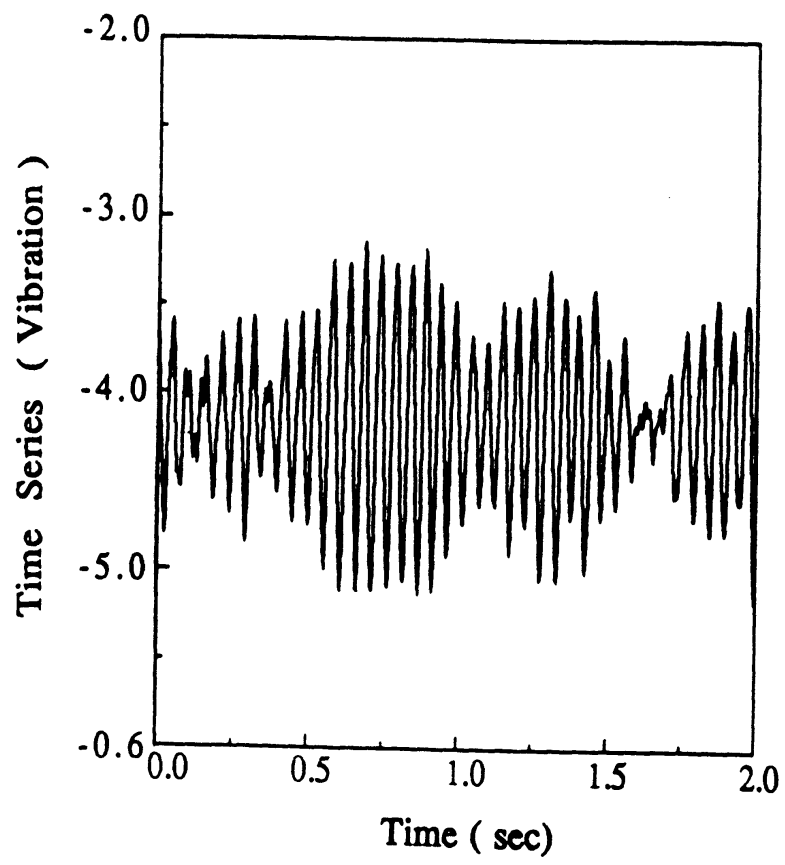

(b)

Figure 10 : The experimental data: (a) Excitation (b) Response.
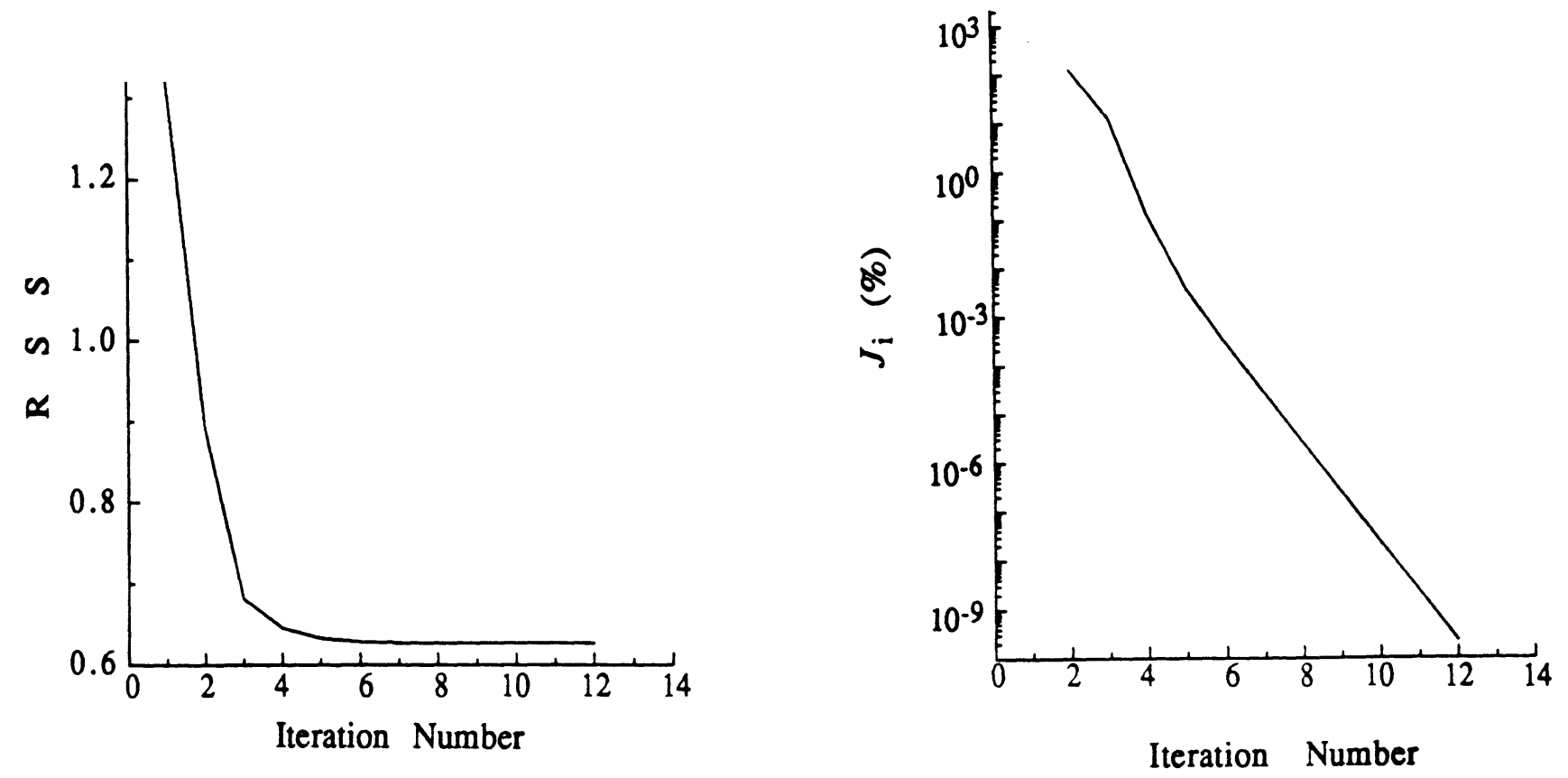

Figure $11:$ The RSS and $J_{i}$ as functions of the iteration number (Beam Experiment). 


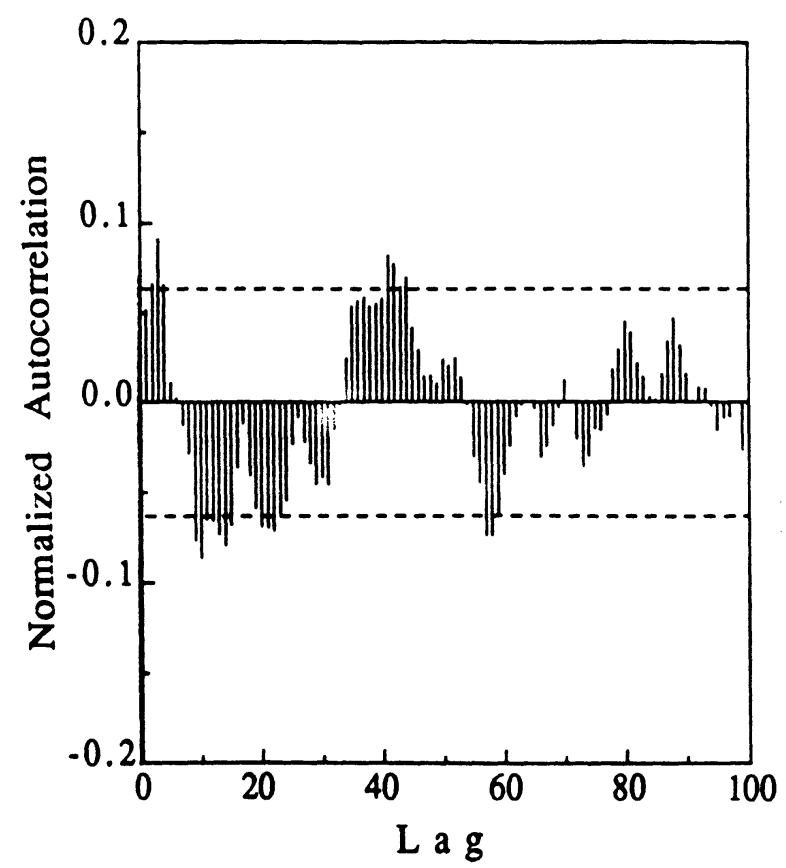

(a)

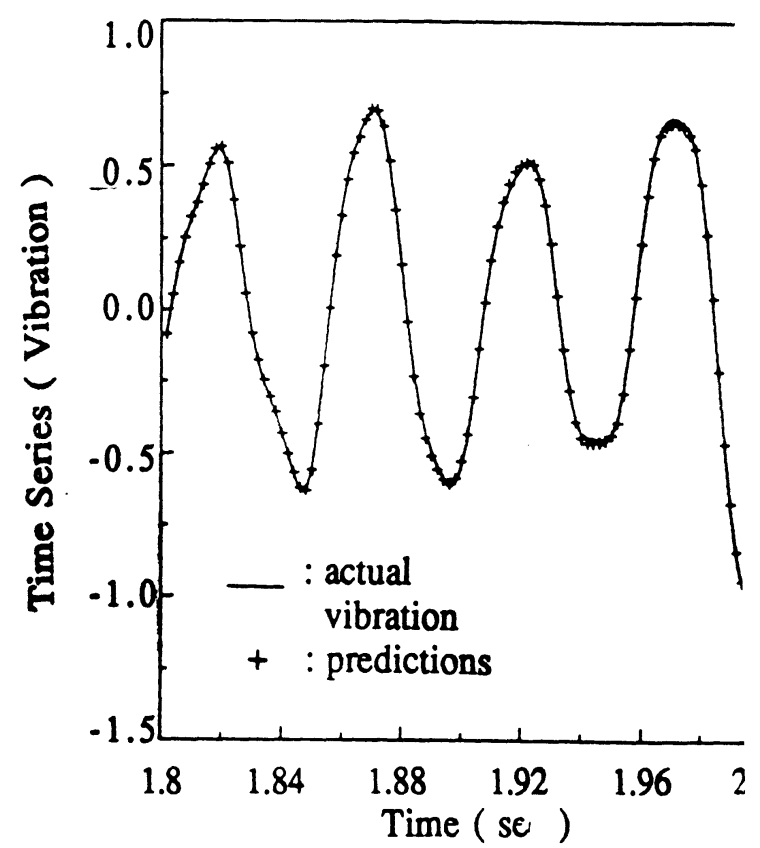

(b)

Figure 12 : Validation of the estimated model: (a) The normalized autocorrelation of the residuals (b) One-step ahead predictions of the vibration signal (Beam Experiment).

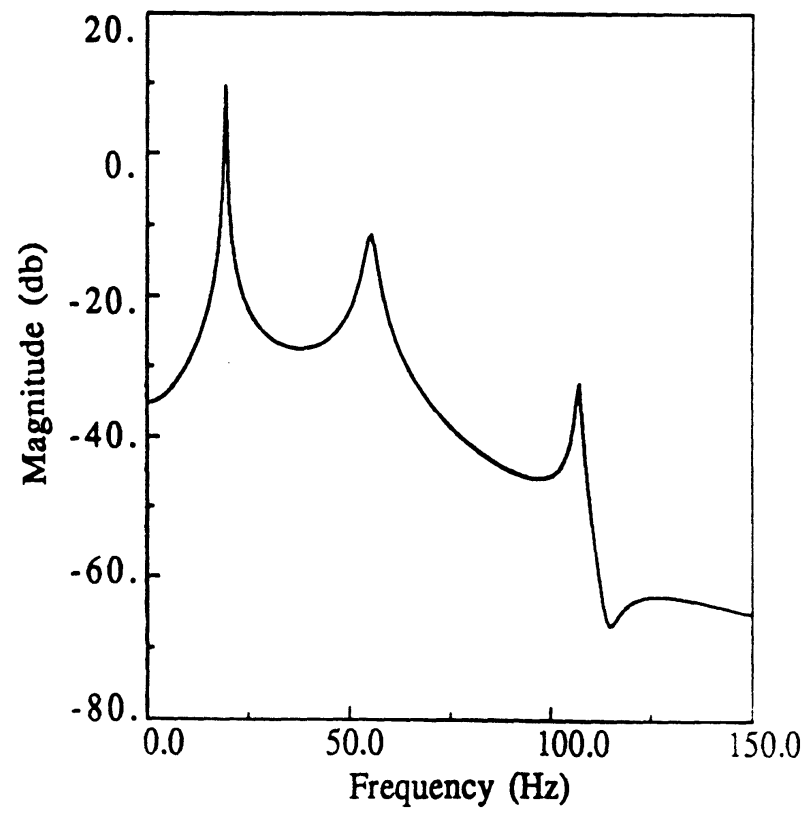

Figure 13 : The frequency response magnitude curve as estimated by the proposed (SML) approach (Beam Experiment). 